\title{
The effects of internal physiology on polyphenic horn development in the dung beetle Onthophagus taurus
}

\author{
Naomi Garrett Williamson \\ West Virginia University, ngw0008@mix.wvu.edu
}

Follow this and additional works at: https://researchrepository.wvu.edu/etd

Part of the Developmental Biology Commons, Entomology Commons, Evolution Commons, Genetics Commons, and the Molecular, Genetic, and Biochemical Nutrition Commons

\section{Recommended Citation}

Williamson, Naomi Garrett, "The effects of internal physiology on polyphenic horn development in the dung beetle Onthophagus taurus" (2020). Graduate Theses, Dissertations, and Problem Reports. 7556. https://researchrepository.wvu.edu/etd/7556

This Thesis is protected by copyright and/or related rights. It has been brought to you by the The Research Repository @ WVU with permission from the rights-holder(s). You are free to use this Thesis in any way that is permitted by the copyright and related rights legislation that applies to your use. For other uses you must obtain permission from the rights-holder(s) directly, unless additional rights are indicated by a Creative Commons license in the record and/ or on the work itself. This Thesis has been accepted for inclusion in WVU Graduate Theses, Dissertations, and Problem Reports collection by an authorized administrator of The Research Repository @ WVU. For more information, please contact researchrepository@mail.wvu.edu. 
The effects of internal physiology on polyphenic horn development in the dung beetle Onthophagus taurus

Naomi Williamson

Thesis submitted to the Davis College of Agriculture, Natural Resources and Design at West Virginia University in partial fulfillment of the requirements for the degree of Master of Science in Genetics and Developmental Biology

\author{
Teiya Kijimoto, Ph.D., Chair \\ Daniel Panaccione, Ph.D \\ Kimberly Barnes, Ph.D \\ Department of Plant and Soil Science
}

Morgantown, West Virginia

2020

Keywords: Metabolomics, Polyphenism, Insect Physiology, Genetics, Development, Evolution, Nutrition

Copyright 2020 Naomi Williamson 


\title{
ABSTRACT
}

The effects of internal physiology on polyphenic horn development in the dung beetle Onthophagus taurus

\author{
Naomi Williamson
}

An organism's phenotype can be affected in development by alterations to gene expression based on environmental inputs. Nutrition is one such environmental input and the central regulator of development of large horn or small horn phenotypes in the dung beetle species, Onthophagus taurus. However, little is known about the nature of chemical compounds that are critical to this plastic horn development. To better understand these compounds, we are utilizing an untargeted metabolomic approach as well as a targeted gene approach. Through the metabolomic approach, it was uncovered that environmental conditions tend to have a greater impact on metabolomic composition than sex. In addition, several discovered metabolites were indicative of different environmental conditions. Under our genetic approach, we discovered that a phosphatidylinositol-4-phosphate (PI4Kiiia) knockdown results in animals having a greater mean horn size, more variance in horn width, and many horn shapes not seen in control animals. 


\section{Acknowledgements}

We would first like to thank Dr. KangMo Ku in the Division of Plant and Soil Sciences for aiding us in our initial metabolomics testing. With his help, GC/MS results were obtained that confirmed that metabolites could be detected and quantified with our current methods.

Next, we would like to thank Dr. Callee Walsh, whose guidance through the metabolomics portion of our project was unparalleled. Dr. Walsh conducted preliminary testing via $\mathrm{LC} / \mathrm{MS}$ as well as the full untargeted metabolomic analysis. She also aided in data interpretation and provided us with clustering analysis.

Finally, we would like to thank Dr. Ida Holaskova, who aided in the statistical interpretation of the results from our genetic approach. Dr. Holaskova provided us with thorough and appropriate analysis that dramatically enhanced the quality of this research project. 


\section{Table of Contents}

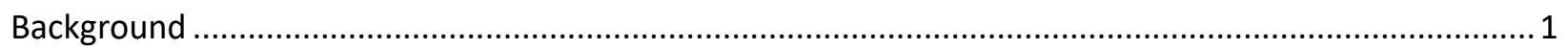

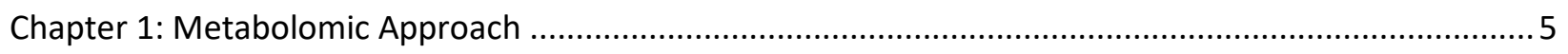

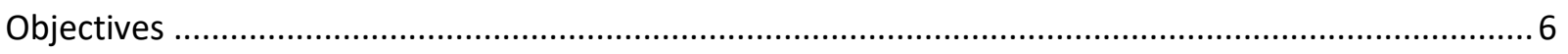

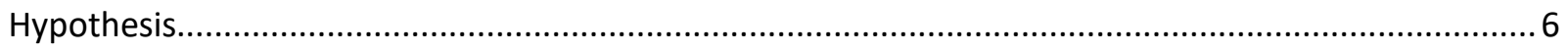

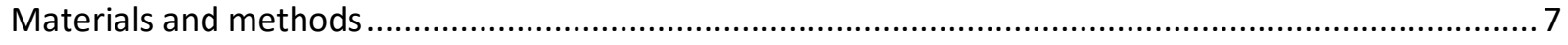

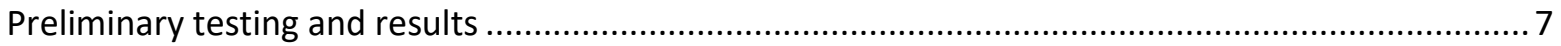

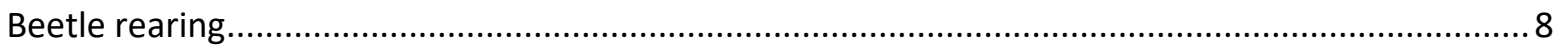

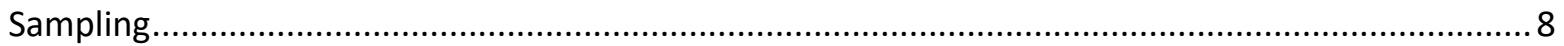

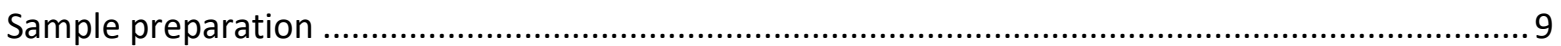

Methanol Extraction of organic compounds from beetle hemolymph ......................................... 9

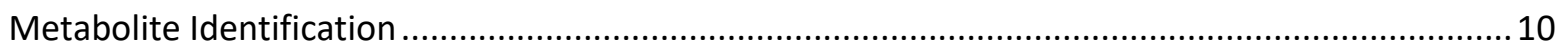

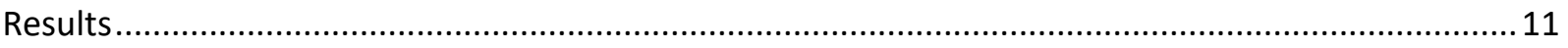

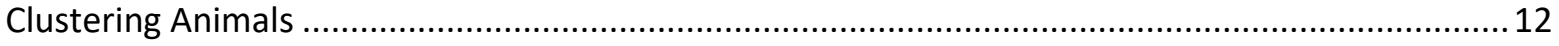

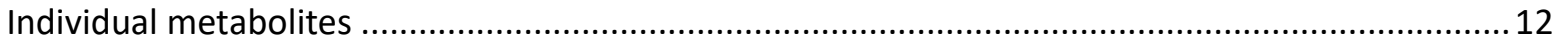

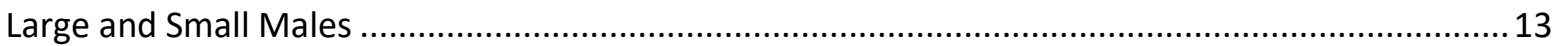

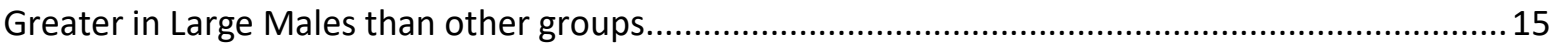

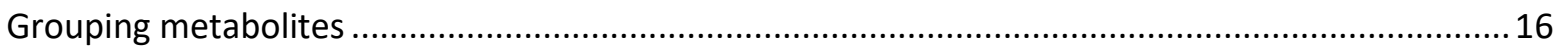

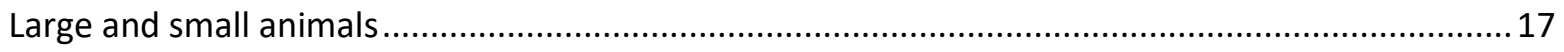

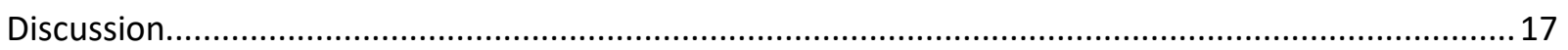

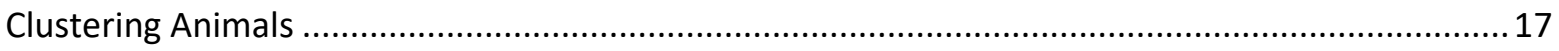

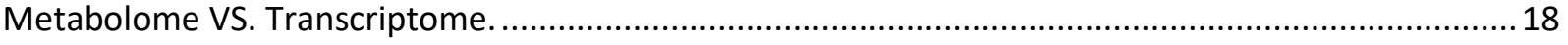

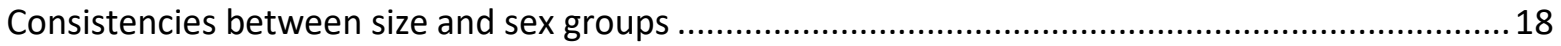

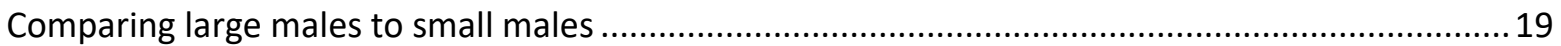

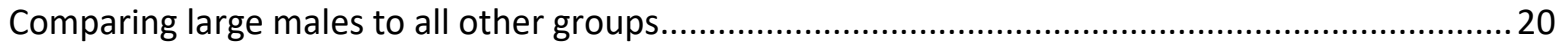

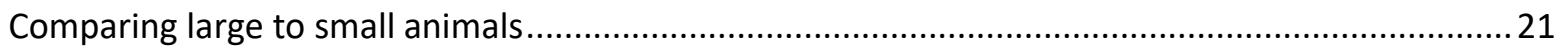

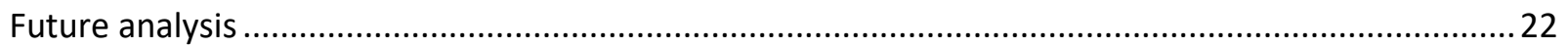

Chapter Two: Exploring the link between PI4P and horn development ............................................23

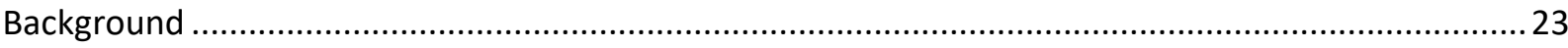

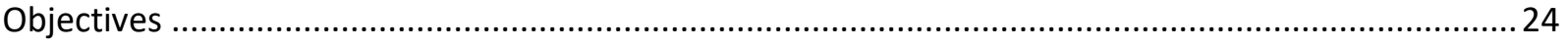

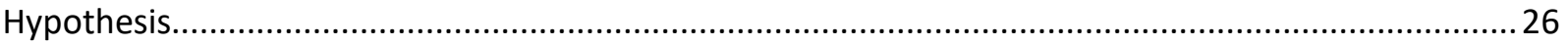

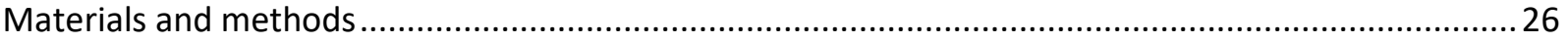

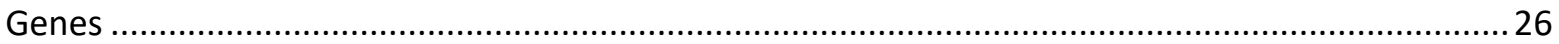


Statistics

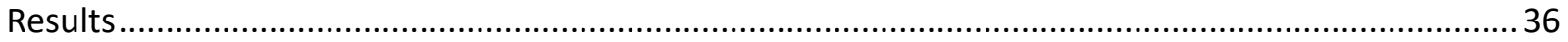

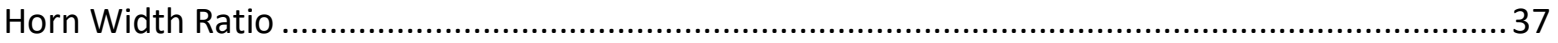

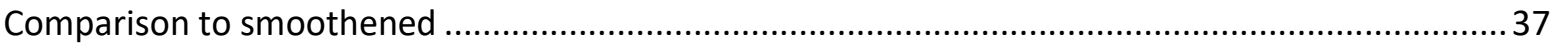

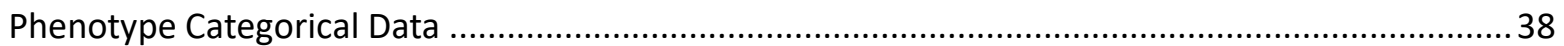

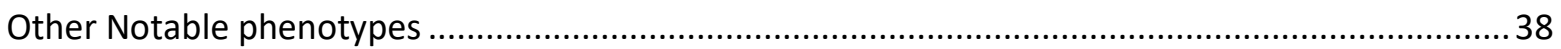

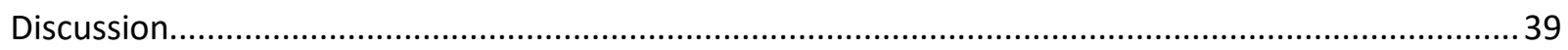

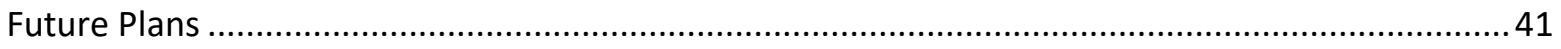

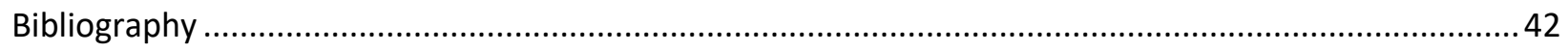




\section{Background}

The term phenotypic plasticity describes an organism's capacity to alter its phenotype in a continuous and linear fashion as a response to changes in environmental conditions, without altering its genotype $(1,2)$. One example of phenotypic plasticity in humans is the plastic response to famine in in utero conditions. Humans exposed to less than optimal nutrient conditions before birth have higher rates of heart disease, schizophrenia, and obesity when compared to those under optimal conditions (3). Zebrafish exhibit temperature-dependent phenotypic plasticity where increased water temperatures lead to the masculinization of female fish, creating a male-female ratio imbalance (4). Polyphenism, on the other hand, is an extreme form of phenotypic plasticity in which two or more distinct morphs can arise, often via threshold mechanics. This process allows organisms with the same genetic background to diverge dramatically depending on environmental conditions.

For instance, male butterflies in the genus Bicyclus display polyphenism through the development of either large eyespots on their wings during the wet season or small ones during the dry season. This is mediated, in part, by differences in hormone titers of 20 hydroxyecdysone (5). Polyphenism is also observed in the water flea Daphnia, where development is affected by the presence of chemical cues released by predators. Under such conditions, Daphnia develop a defensive morph featuring neck teeth. Otherwise, they develop into adults with less defensive phenotypes and no neck teeth (6).

In order to better understand how organisms respond to different environmental conditions through polyphenism, we utilized the dung beetle Onthophagus taurus. More 
specifically, we study how the environmental factor nutrition affects polyphenic horn development. This species serves as an excellent model for understanding polyphenism, as males develop into one of two distinct phenotypes in response to nutritional input. The large horned phenotype develops in response to optimal nutrient conditions, whereas the small horned phenotype develops in response to sub-optimal conditions (7). While horns in O. taurus are considered polyphenic, body size (thorax width) operates under phenotypic plasticity, with higher quantity and quality nutrients leading to larger body sizes (fig. 1).

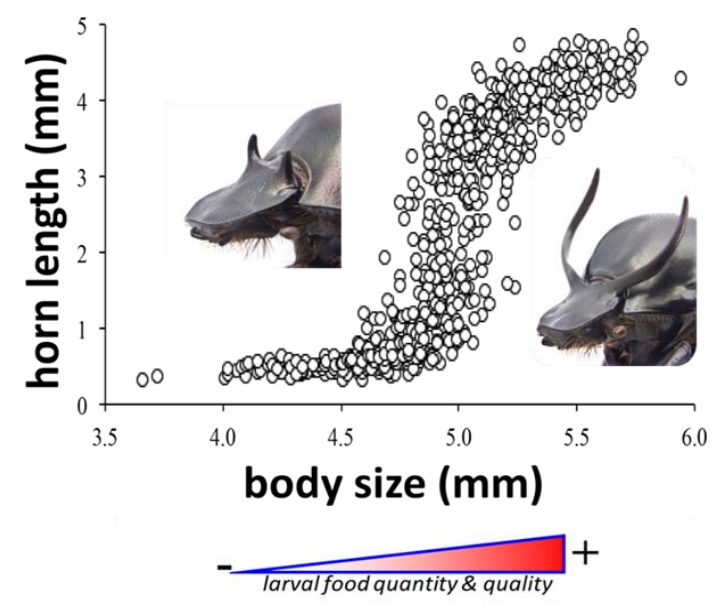

Figure 10 . taurus horns and body sizes (thorax widths) are nutritionally dependent. Body size increases in a linear fashion with food quantity and quality. Horns sizes are distributed in a bimodal fashion and have one of two extreme phenotypes

Large males typically outcompete their smaller counterparts in the form of horn-to-horn combat. However, small males take on a less aggressive, sneaking behavior to find mating partners. In the case of $O$. taurus, evolution favors extreme phenotypes. Males with 
intermediate horn phenotypes are less fit than the other morphs and, therefore, are typically less common in natural populations (8).

Whether small, large, or the uncommon intermediate, horns in beetles are considered a novel structure as they appear to lack homology with other organs in the individual as wells as with organs in other insect groups. The horns of $O$. taurus take up a significant portion of their body mass and are often longer than other appendages (9).

Organisms can evolve novel structures by repurposing already established genes and pathways through a process known as genetic co-option. Genetic co-option results when gene regulation is altered to form new developmental or physiological traits while the gene's original function is maintained (10).

Among the developmental processes that contribute to horn development, our lab currently focuses on those involving doublesex and hedgehog, as they have been suggested to be co-opted for horn development $(8,11)$. The gene doublesex $(d s x)$ produces male and female mRNA isoforms through alternative splicing that result in different transcription factors between the sexes (12). In 0 . taurus, dsx maintains its ability to establish sexual dimorphism, however, it has also been co-opted to mediate nutrition-dependent horn development in males and suppress horn development in females (11).

The focus of this project is another co-opted developmental process that is regulated by the gene hedgehog $(h h)$, The Hh pathway is a highly conserved developmental pathway in bilaterians and is necessary for appendage development in insects $(13,14)$. The protein $\mathrm{Hh}$ is secreted in a gradient, establishing the patterning of the anterior-posterior axis within 
segments and appendage primordia (such as wings and legs) during development $(8,15)$. This gradient is, in part, controlled by modification of the Hh protein by cholesterol on the Cterminus and palmitate on the $\mathrm{N}$-terminus (16).

Our working model is that $\mathrm{Hh}$ and $d s x$ regulate nutritionally-dependent horn development in $O$. taurus. Under high nutrient conditions, $d s x^{\prime} s$ co-opted role is to stimulate the development of large horns, while the Hh pathway contributes to horn repression under low nutrient conditions $(8,11)$.

In addition to these two developmental processes, insulin signaling also contributes to horn development. The highly conserved insulin signaling pathway mediates nutritiondependent growth and nutritional sensitivity in insect organs and appendages, partially through the growth inhibitor, Foxo. In a study by Casasa et al. 2018, Foxo was knocked down, resulting in small males having larger horns than control animals. These results suggest that this gene is partially responsible for nutritional-dependent horn polyphenism and horn suppression in small males. Current literature proposes that Foxo promotes Smoothened (Smo), a crucial component of Hedgehog signaling, under low nutrient conditions in wild type organisms. Another component of the pathway, insulin receptor 2 (InR2), was found to promote $d s x$ expression and, thus, horn growth under high nutrient conditions (17). Interestingly, the specific nutritional factors contributing to the activation and suppression of these pathways remains relatively unknown. Another unknown factor is how 0 . taurus processes nutrients from the environment that contribute to horn polyphenism. 
In order to better understand this, two approaches have been taken in this project. First, an unbiased metabolomic approach was utilized to determine the makeup of the internal nutrient environment of four beetle groups: large male, small male, large female, and small female. Our primary objective was to obtain an extensive list of metabolites and determine the differences between the groups. By determining which metabolites are present in each beetle group, a better understanding of which nutrients aid in the activation or suppression of horns can be gained.

Simultaneously, a genetic approach has been taken to better understand the role of the gene PI4KIII $\alpha$, on a specific metabolite, phosphatidylinositol-4-phosphate (PI4P), which we propose is involved in horn development through the Hedgehog pathway. By studying this gene, a better understanding of a specific metabolite's role in horn development was expected to be gained.

\section{Chapter 1: Metabolomic Approach}

In order to visualize the metabolomic similarities and differences between groups of beetles, the metabolome was analyzed. While metabolites are substances that typically have low molecular weights and are reactants, intermediates, and products of biochemical reactions, the metabolome refers to all of the metabolites in a biological sample at a given point in time (18). Examples of metabolites include phospholipids, steroids, amino acids, carbohydrates, and fatty acids (18). They can also act as signaling molecules and activate or repress certain genes or pathways. For example, the metabolite focused on in the targeted gene approach, 
phosphatidylinositol-4-phosphate (PI4P), can act as a signaling molecule in the Hedgehog pathway by donating a phosphate to the protein, Smoothened (Smo) (19).

Two strategies are commonly utilized to generate metabolomic data: targeted and untargeted (also known as biased and unbiased, respectively) approaches. Targeted metabolomics focuses on specific metabolites with known structures, while the goal of untargeted metabolomics is to identify and measure as many metabolites as possible $(20,21)$. The untargeted approach is useful for generating hypotheses and discovering relative metabolite quantities (20).

\section{Objectives}

The primary objective of this project was to obtain untargeted metabolomic data from O. taurus of different sexes and nutritional conditions. Through this approach, differing levels of metabolites among groups can be highlighted and further insight can be gained surrounding the physiological reaction of beetles in response to different environmental inputs. The end goal is to determine what metabolites play a role in the development of horns in large males. Prior to this project, no progress has been made analyzing the $O$. taurus metabolome.

\section{Hypothesis}

The external environment that a beetle develops in will affect the beetles' internal physiology, leading to observable changes in phenotype. Therefore, we hypothesize that environmental factors and genetic underpinnings will result in different metabolite composition and/or level among different groups of beetles. We hypothesize that beetles will share more metabolomic similarities within groups than they will with beetles from other groups. 


\section{Materials and methods}

\section{Preliminary testing and results}

Before global untargeted testing, preliminary analyses were conducted using Gas Chromatography Mass Spectrometry (GC/MS) and Liquid Chromatography Mass Spectrometry (LC/MS) methods. LC/MS is considered more suitable for analyzing metabolites, as it can sense non-volatile compounds as well as compounds at higher molecular weights. Therefore, it was chosen for the full global analysis.

As part of preliminary testing, GC/MS was utilized to determine if our current methods of metabolite extraction were viable. These initial results concluded that there was a significant difference in the amount of cholesterol present in the large male and female beetles, thus establishing that organic compounds were able to be extracted, detected, and quantified in $O$. taurus hemolymph on a mass spectrometer (fig. 2).

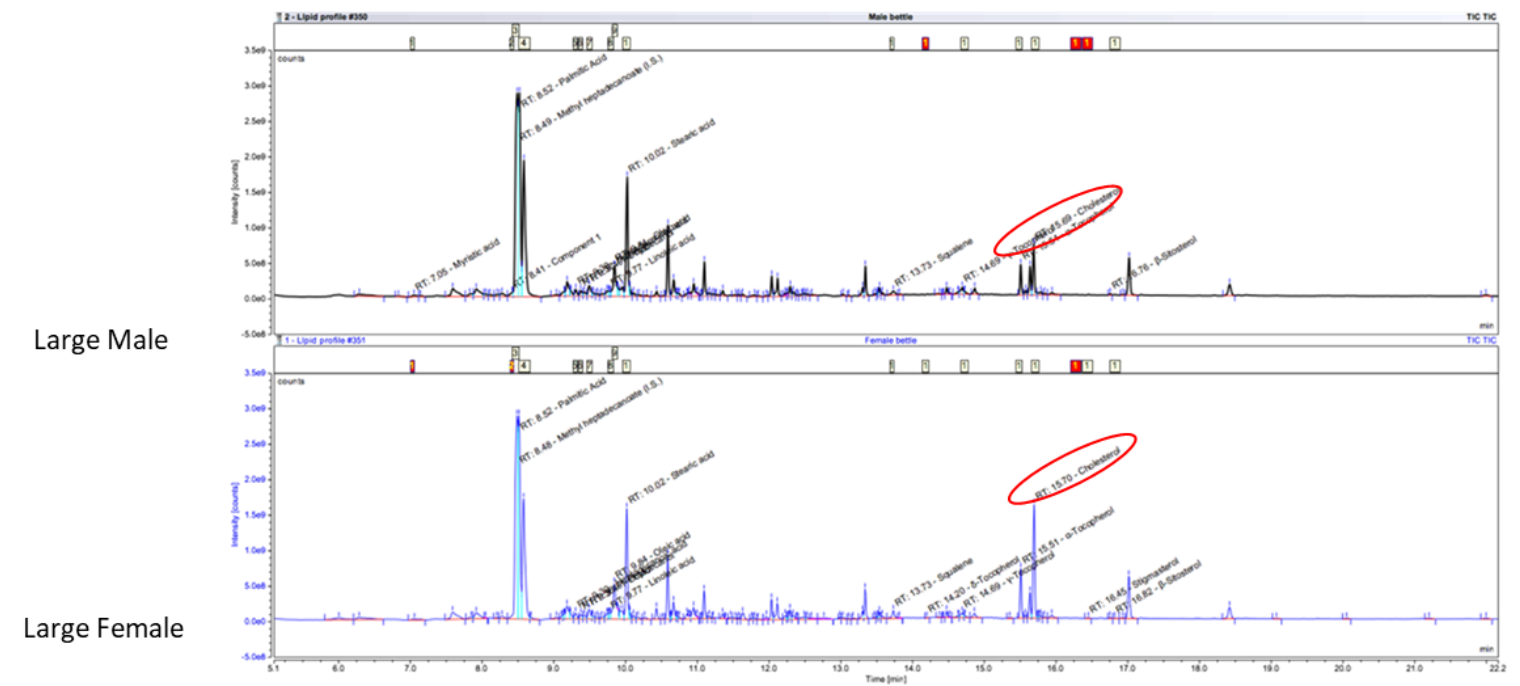

Figure 2: GC/MS result from one large male and one large female beetle. Cholesterol (circled in red) is higher in the female than the male. This preliminary test established that metabolites were able to be identified and quantified using our current met 


\section{Beetle rearing}

Beetles were collected at WVU's Animal Sciences research farm in Morgantown, WV and used for breeding purposes. Offspring were reared under laboratory conditions at $26^{\circ} \mathrm{C}$ in a regulated 16-hour light and 8-hour dark cycle. Dung was collected at the farm and homogenized to ensure beetles would have relatively equally nutritious dung. Larvae were transferred from their brood balls to plates with homogenized transfer dung and kept in an incubator at $26^{\circ} \mathrm{C}$ in the same light-dark cycle.

\section{Sampling}

As with other beetle species, all 0 . taurus undergo complete metamorphosis with an egg, larval, pupal, and adult stage. Until they reach adulthood, dung beetles remain in individual brood balls that are produced by their parents. The chosen time point for this study occurs during the larval stage and immediately before pupation. More specifically, animals were obtained around 48 hours after they enter the prepupa stage (referred as PP2 hereafter). During their PP2 stage, beetles appear opaque white and are immobile. They have completed all pre-adult feeding and their horn sizes have already been established due to the quantity and quality of their brood balls. Thus, body mass during PP2 is indicative of future adult body and horn size.

Beetles were collected from the incubator during PP2 stage and rinsed briefly in 70\% methanol. They were then measured for body mass, flash frozen using liquid nitrogen, and stored at $-80^{\circ} \mathrm{C}$. Samples were collected between September 2018 and March 2019. Due to the 
consistency of laboratory conditions, insects were not affected by the time of the year they were collected.

Sample preparation

Five beetles were selected from each group for testing. Large males $(0.1458,0.1477$, $0.1485,0.1491,0.1492 \mathrm{~g})$, small males $(0.0773,0.0905,0.0934,0.1003,0.1008 \mathrm{~g})$, large females $(0.1374,0.143,0.1435,0.1469,0.1568 \mathrm{~g})$, and small females $(0.0885,0.09,0.0925,0.0954$, $0.1032 \mathrm{~g}$ ) were selected for analysis. However, it is important to note that one of the large male samples was misplaced during preparation and had to be excluded from the analysis.

\section{Methanol Extraction of organic compounds from beetle hemolymph}

Organic compounds were extracted from beetles using a cold methanol extraction method, due to its efficacity and ability to extract a broad spectrum of metabolites at once (20). This is especially beneficial, as future hypotheses can be more easily generated.

Beetle larvae were kept on ice during the extraction process. Hemolymph was obtained by centrifugation at $8000 \mathrm{rpm}$ for 15 minutes at $4{ }^{\circ} \mathrm{C}$. To extract hemolymph, a small hole was made at the bottom of a tube (referred as the sample tube hereafter) containing the beetle larva using a needle. Sample tubes were then placed on top of collection tubes before centrifugation. Centrifugation separated hemolymph from insoluble debris and further accumulated it into soft pellets in the collection tube. Sample tubes were removed from the collection tubes and discarded. Soft pellets were then resuspended and centrifuged again to ensure the samples were well mixed. Equal amounts of supernatant were removed from each tube, moved into extraction tubes, and labeled accordingly. Ice cold methanol was added in a 
3:1 volume ratio to each sample. Five quality control samples were included and contained equal amounts of pooled hemolymph from each beetle. Fifty $\mu \mathrm{M}$ of internal standard (caffeine and denatured amino acids) were added to each beetle and quality control sample. Samples were then sonicated for five minutes and transferred to the $-20^{\circ} \mathrm{C}$ freezer for one hour.

Liquid chromatography mass spectrometry (LC/MS) was performed using HILIC chromatography in negative ion mode on each beetle and QC sample. Ten mM ammonium acetate in water made up aqueous buffer (mobile phase) and 100\% acetonitrile was the organic buffer (mobile phase). Using a Q Exactive Orbitrap mass spectrometer (Thermo Scientific), $\mathrm{ms} / \mathrm{ms}$ data were collected in full scan mode over the range of $\mathrm{m} / \mathrm{z} 100$ to 1000 . Ms/ms spectra data were also collected from QC samples to aid in identification. Data was then processed utilizing Compound Discoverer (version 3.0; Thermo Scientific).

\section{Metabolite Identification}

Mass data $(\mathrm{m} / \mathrm{z})$ from LC/MS was quarried against the databases Chemspider and Metabolika to predict identities with a mass tolerance of up to $5 \mathrm{ppm}$ (22). Metabolite identities were further supported by $\mathrm{ms} / \mathrm{ms}$ by selecting $\mathrm{m} / \mathrm{z}$ values from fragmented ions and comparing them to libraries of $\mathrm{ms} / \mathrm{ms}$ spectra. Candidate metabolite names were identified by referring to $\mathrm{mzCloud}$ database given $\mathrm{m} / \mathrm{z}$ values with a mass tolerance of up to $5 \mathrm{ppm}$ (22). An ANOVA test was utilized to determine statistically significant changes ( $p$-value $<0.05$ ) between the four treatments (large male, small male, large female, small female) at any fold change (fig. 3). 


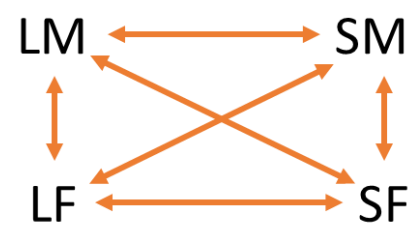

Figure 3: Metabolite levels were compared between all groups.

Orange arrows represent

Results

Through global unbiased testing, 1031 metabolites were discovered. 241 of these were identified by mass by at least one database (Chemspider or Metabolika), while 49 were further identified through fragmentation data by $\mathrm{ms} / \mathrm{ms}$ and the $\mathrm{mzCloud}$ database.

An ANOVA was utilized to detect differences between the various groups (table 1). Of the 1031 metabolites discovered, 371 had a significant difference between two or more groups.

While the large male and small male comparison had the most significantly different (alpha <

Table 1: Number of significantly different metabolites for each group comparison. The largest number of differences can be found between the large male and small while the small male and small female have fewest. number of differences.

\begin{tabular}{|l|l|l|l|}
\hline & $\begin{array}{l}\text { Total metabolite } \\
\text { differences }\end{array}$ & $\begin{array}{l}\text { Number of metabolites } \\
\text { with higher levels in } \\
\text { first group }\end{array}$ & $\begin{array}{l}\text { Number of metabolites } \\
\text { with higher levels in } \\
\text { second group }\end{array}$ \\
\hline $\begin{array}{l}\text { Large Male v Small } \\
\text { Male }\end{array}$ & 148 & 112 & 36 \\
\hline $\begin{array}{l}\text { Large Female v Large } \\
\text { Male }\end{array}$ & 31 & 3 & 28 \\
\hline $\begin{array}{l}\text { Small Female v Large } \\
\text { Male }\end{array}$ & 85 & 20 & 65 \\
\hline $\begin{array}{l}\text { Large Female v Small } \\
\text { Female }\end{array}$ & 40 & 20 & 20 \\
\hline $\begin{array}{l}\text { Large Female v Small } \\
\text { Male }\end{array}$ & 44 & 22 & 22 \\
\hline $\begin{array}{l}\text { Small Female v Small } \\
\text { Male }\end{array}$ & 21 & 14 & 7 \\
\hline
\end{tabular}


0.05 at any fold change) metabolites, the small female and small male comparison had the least number of differences (alpha $<0.05$ at any fold change).

\section{Clustering Animals}

The first goal was to cluster animals based on metabolomic similarities in order to determine consistencies within groups. A hierarchical model with Pearson Complete clustering was chosen due to this model's scale invariance. Animals tended to cluster more closely based on size rather than sex (fig. 4).

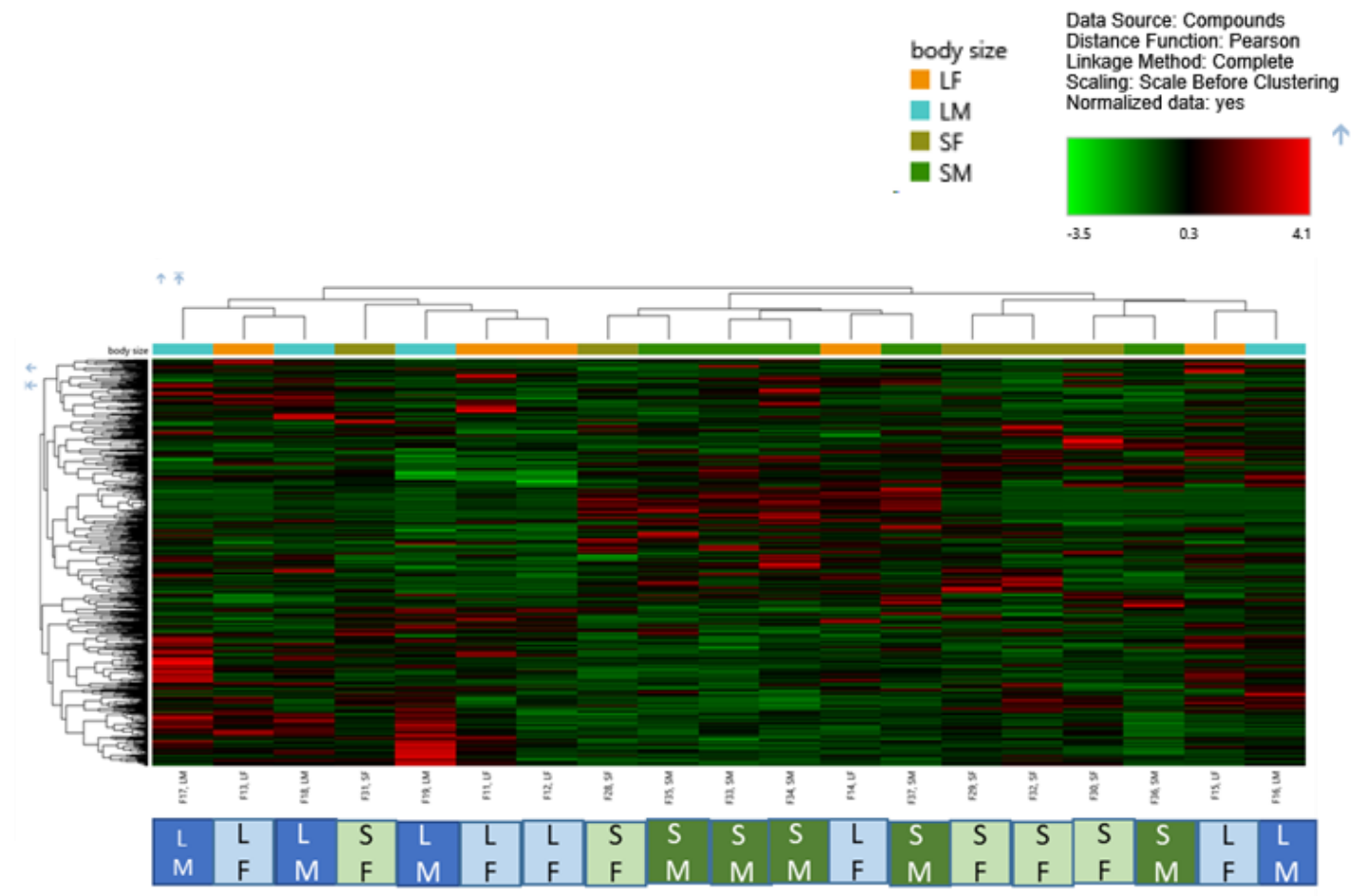

Figure 4: Hierarchical clustering of beetles based on metabolomic similarities. Animals tend to group together based on size rather than sex. This indicates that the external environment impacts the metabolome more than sex.

Individual metabolites

Next, we aimed to identify individual metabolites that may be products or intermediates of pathways that contribute to phenotypic differences or result from differently nutritious 
environments. When listing potential metabolites for analysis, we defined a criterion of being:

a) significantly different in a relevant comparison and b) having a biochemical function relating to our project. While ranking metabolites, those that were identified by fragmentation and quarried against a library ( $\mathrm{ms} / \mathrm{ms}$ ) were ranked first. One metabolite not identified by fragmentation (D-Xylonic acid) was included due to it's increased significance, function that correlates with our project, and identification through mass (Chemspider, Metabolika).

\section{Large and Small Males}

We aimed to identify metabolomic differences between large and small males. As shown in table one, most of the metabolite differences were between the large and small male comparison and most metabolites were greater in the large male than the small male. Of the 1031 metabolites discovered, 148 of them were significantly different (alpha >0.05) in the large and small male group at any fold change. Thirty-four of these significantly different metabolites were named by quarrying mass data against Chemspider and Metabolika and 5 were further identified using fragmentation data in $\mathrm{ms} / \mathrm{ms}$ (fig. 5). 


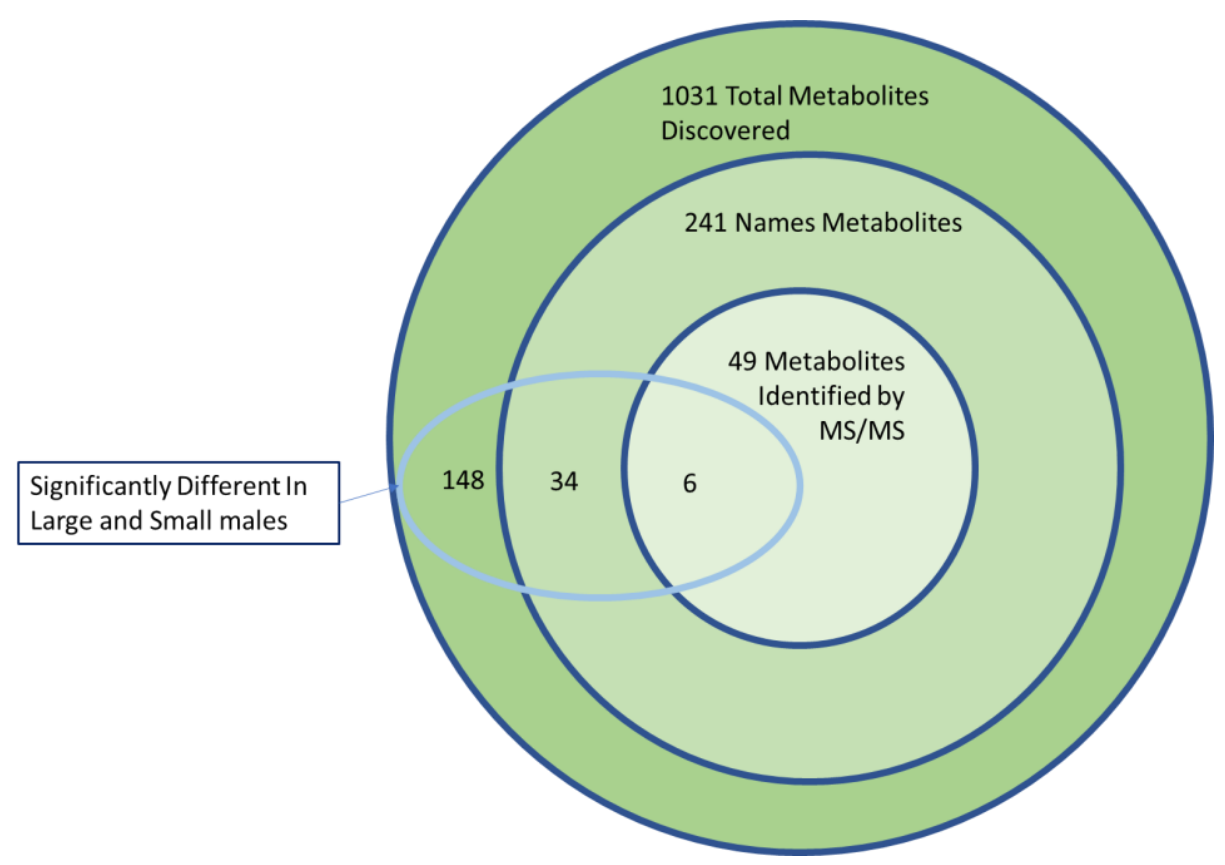

Figure 5: Overall and significantly different numbers of discovered metabolites, named metabolites, and metabolites identified by $\mathrm{ms} / \mathrm{ms}$. Of the 1031 metabolites discovered, 148 were different in the large male/small male comparison.

Of the 5 metabolites that were identified by fragmentation through $\mathrm{ms} / \mathrm{ms}$ and were significantly different between large and small male comparison, 3 were uniquely different in this comparison. These include $\mathrm{N}$-acetyl-DL-glutamic acid, glycerol 3-phosphate, and citric acid. The others two included N-Acetyl-L-glutamine and D-Glutamine, and were also significantly different in the large female and large male comparison as well as the small female and large male comparison.

Citric acid is a metabolite identified by fragmentation through $\mathrm{ms} / \mathrm{ms}$ and is 1.5 times (log2 fold change-0.38) significantly greater in large males than small males (fig. 6). Glycerol-3phosphate was identified through fragmentation data by $\mathrm{ms} / \mathrm{ms}$ and indicated as significantly 
greater in large males than small males by 2.1 times. N-acetyl-DL-glutamic acid (N-

acetlyglutamate) was identified through fragmentation data by $\mathrm{ms} / \mathrm{ms}$ and was significantly

greater in small males than large males by 1.80 times.

\section{Greater in Large Males than other groups}

The next goal was to determine what metabolites are significantly higher in large males than in the other three groups (large female, small female, and small male). By doing so, metabolites hypothetically involved in horn development may be identified. Ten metabolites were significantly different in large males than the other groups including D-(-)-glutamine, Nacetyl-L-glutamine, Y-aminobutyric acid (GABA), epsilon-(gamma-Glutamyl)-lysine, and pyroglutamine.

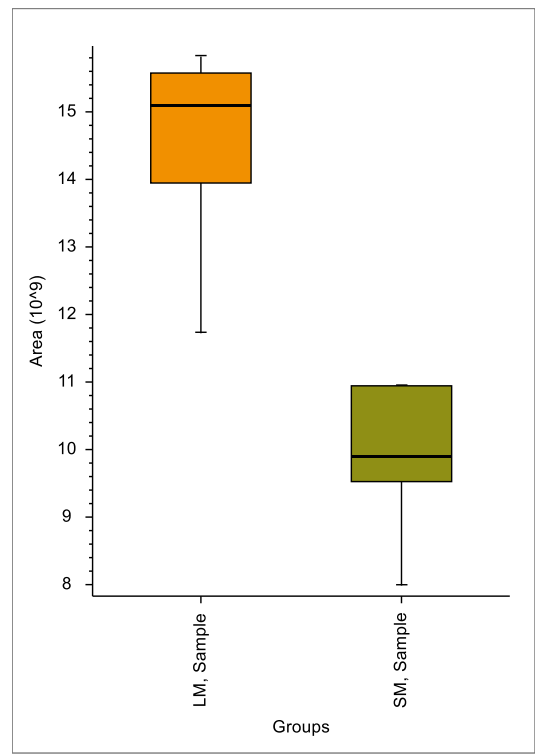

Figure 6: Citric acid is a metabolite identified by $\mathrm{ms} / \mathrm{ms}$ that is higher in large males than small males. This metabolite may result from beetles developing in an environment with higher nutrient conditions.

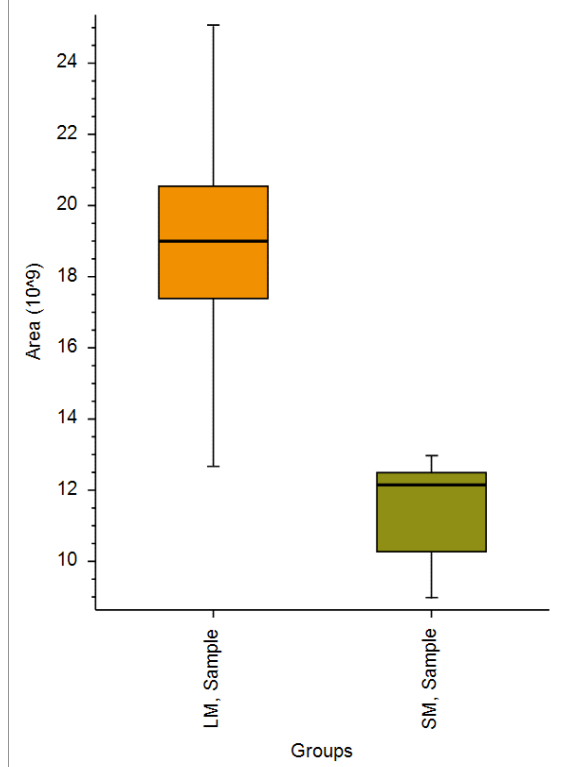

Figure 7: D-Glutamine is a metabolite identified by $\mathrm{ms} / \mathrm{ms}$ that is significantly higher in large males than any other beetle group. This metabolite may be the biproduct of a nutrient rich environment. 
Of the 10, two metabolites (D-(-)-Glutamine, N-Acetyl-L-glutamine) were identified through fragmentation by $\mathrm{ms} / \mathrm{ms}$. D-Glutamine is a potential indicator of a high nutrient environment while $\mathrm{N}$-Acetyl-L-glutamine is a derivative of glutamine.

D-Glutamine is a metabolite identified by fragmentation through $\mathrm{ms} / \mathrm{ms}$ and is 1.57 times significantly greater in large males than small males, 1.48 greater than in small female, and 1.40 times greater than in large female (Fig. 7). N-Acetyl-L-glutamine and is 2.38 times significantly greater in large males than small males, 2.87 greater than in small female, and 2.04 times greater than in large female.

\section{Grouping metabolites}

The next goal involved grouping metabolites from comparisons to determine how many metabolites, as well as what kinds of metabolites, were found to show overlap between groups.

Out of metabolites that significantly different at any fold change, 9 were similarly different between males and females of the same size. 9 of the 28 (32\%) metabolite differences

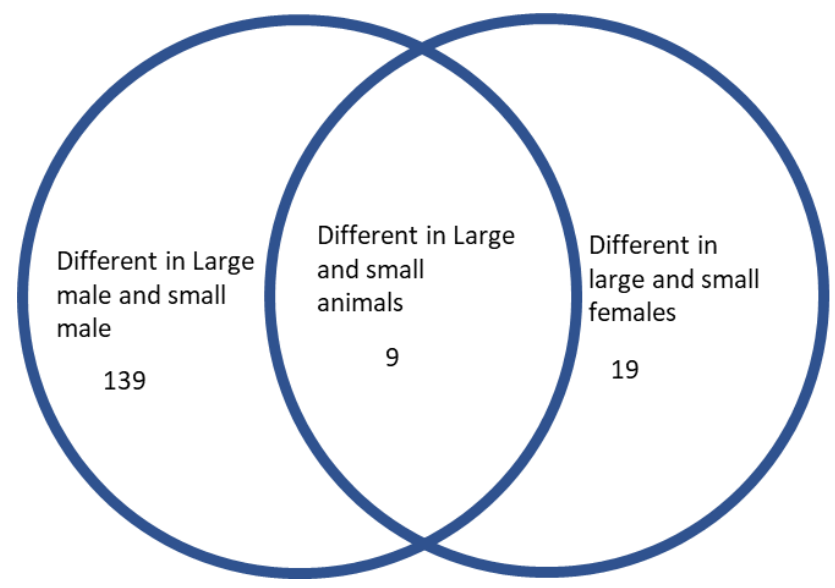

Figure 8: Differences in the male groups, female groups, and overlapping metabolites that behave similarly in both groups. 
between large and small female are similarly different in large and small males (9 of 149 (6\%)). Metabolites that are similarly present in each size group are likely the result of similarities in nutrient conditions (fig. 8).

\section{Large and small animals}

As mentioned, 9 metabolites were shared between the male comparison (large vs small) and female comparison (large vs small). D-Xylonic acid, is one such metabolite that has not been identified by $\mathrm{ms} / \mathrm{ms}$, however, it was identified by mass through the Metabolika and Chemspider databases. D-Xylonic acid is found to be significantly higher in small males than large males by 5.78 times and 3.7 times greater in small females than large females.

\section{Discussion}

Prior to this project, no known information on the 0 . taurus metabolome had been published. From this study, we gained a better understanding of the scope of metabolites that can be gained from methanol extraction on 0 . taurus hemolymph, as well as what types of metabolites may need to be analyzed using another approach.

\section{Clustering Animals}

One of the most important findings is that animals tend to cluster more closely with regards to size rather than sex. This suggests that the external environment has a bigger impact on the metabolome than the underlying genetic coding for sex. Interestingly, these findings supplement a transcriptome analysis by Kijimoto et al., where sex contributes more to overall variation than nutrition (23). This is potentially due to the metabolome being primarily 
influenced by the environment, while the transcriptome is mainly associated with genetic underpinning.

\section{Metabolome VS. Transcriptome.}

Animals that grouped away from their size and sex groups may have been selected during slightly different time points within the PP2 stage, which lasts roughly 24 hours. Throughout this time period, the metabolite composition may change as beetles approach their molting phase. This may have led to issues, as individuals were not collected at the same time point during PP2. Future researchers may benefit from developing a protocol that allows them to select all beetles at the same point of time to reduce variabilities.

\section{Consistencies between size and sex groups}

Our suggestion that size has more influence on metabolomic composition than sex is further supported by results from metabolite grouping. For this goal, we listed all of the metabolites that differed among the large male and small male groups and compared that list to all of the metabolites that differed among the large female and small female groups. This allowed us to compile a list of overlapping metabolites that were significantly different in the large female and large male groups compared to the small male and small female groups. We discovered 9 metabolites that were equally different among the groups. These metabolites were sex independent and size dependent. We speculate that these nine metabolites are nutrition dependent, as they are shown to differ equally among the sexes.

To supplement this, we aimed to determine overlapping metabolites that changed due to sex but were not affected by size. For this approach, we compared a list of metabolites that 
were different in the large male and large female to a list of metabolites that were different in the small male and the small female groups. Overlapping metabolites are expected to be sex dependent and size independent. Overall, no metabolites were found in this comparison. This leads us to speculate that there are very few metabolites related to sex, or, that they cannot be identified using our current methods.

\section{Comparing large males to small males}

Several specific metabolites such as citric acid are greater in large males than small males but show no significant changes in any other group comparison. The metabolite citric acid is an intermediate in the Krebs cycle and indicates a more nutrient rich environment. Citric acid can also be converted into acetyl-CoA for fatty acid synthesis, perhaps another result of taking in excess nutrients. However, fatty acid was not observed in this study. At high levels, citric acid inhibits phosphofructokinase, signaling a decrease in glycolysis. This is due to high levels of citric acid being indicative of abundant biosynthetic precursors, thus, the organism has reduced need for glycolysis (24). Taken together, this information provides evidence that citric is an indicator of large males having a more nutrient rich environment than the small males.

Another metabolite that is only significantly higher in large males than small males is glycerol 3-phosphate. It can be synthesized from glycerol, converted to dihydroxyacetone phosphate (DHAP) followed by a conversion to glyceraldehyde 3-phosphate (GA3P) where it can enter into glycolysis. This increase in glycerol 3-phosphate indicates mobilization of fats (which are stored as triglycerides) in large males where energy is needed to form horns during pupation (25). 
However, glycerol 3-phosphate could also be involved in fatty acid synthesis rather than mobilization. When fatty acids are synthesized in conditions of excess energy input, they are esterified to glycerol 3-phosphate and stored as triglycerides (26).This would indicate a high nutrient environment, where large males are able to store more energy than their small male counterparts.

Both of these metabolites signify an increase in energy in the large male over the small male. While we suspect that an increase in glycerol 3-phosphate may result from mobilization of stored lipids, high citric acid may mean more activity in the Krebs cycle. This implied increased in energy indicates heighted cell growth and, thus, large horn development.

$\mathrm{N}$-acetyl-DL-glutamic acid is another metabolite that met our criteria; however, it was higher in concentration in small males than in large males. This metabolite is synthesized from glutamate which is synthesized from glutamine (27). N-acetlyglutamate is also a positive regulator in the urea cycle. Higher urea cycle activity may signify an increased breakdown of amino acids in small males when glucose is not readily available (28).

\section{Comparing large males to all other groups}

D-Glutamine may also be indicative of a beetle developing in a nutrient rich environment. This metabolite, however, is significantly greater in the large male group than any other groups.

Glutamate, which is synthesized from glutamine, has been recognized as a key metabolite for insect cell growth. One study utilized insect cell lines to determine activity under different conditions, including glutamine free conditions. This study found that cells that mainly 
utilized glutamate grew faster than cells that utilized mainly glutamine (29). These results may provide evidence that higher levels of glutamine predict high nutrient conditions in insects. Our study shows higher levels of glutamine in large insects. This suggests that these insects have enough glutamate in their environment to spare glutamine.

Glutamine is synthesized to glutamate and then $\alpha$-ketoglutarate through a process known as glutaminolysis, and it can be used to replenish the $\alpha$-ketoglutarate intermediate in the Krebs Cycle (30). Taken together with an increase in citric acid, these results could imply that there is more Krebs cycle activity in large males than in small males, and possibly, females.

$\mathrm{N}$-acetyl-L-glutamine is another metabolite identified by fragmentation data through $\mathrm{ms} / \mathrm{ms}$ and seen in higher levels in large males than any other group. This metabolite is a derivative of glutamine.

\section{Comparing large to small animals}

Other metabolites may be indicative of a lower quality nutrient environment or processes suppressing horn development. D-xylonic acid is a metabolite that is found in significantly higher quantities in small animals (male and female) than large. This sugar acid contains a saccharide unit and is derived from xylitol (31). Due to its relationship with xylitol, this metabolite indicate that small animals are taking in more low nutrient plant matter such as straw.

These results provide evidence that size affects the metabolome more than sex and that several of the discovered metabolites are indicators of a beetle's nutrient environment and its effect on the beetle's phenotype. Other discovered metabolites may be byproducts or 
intermediates of processes influencing horn development under different nutrient conditions. By obtaining an extensive list of metabolites, this project has laid the groundwork for future endeavors combining nutrition, genetics, and resulting phenotype in 0 . taurus.

\section{Future analysis}

For future analysis, chloroform may be utilized to further extract more non-polar metabolites from samples previously extracted with methanol. This method could potentially be based on the Bligh-Dyer Method for lipid extraction which combined methanol and chloroform extraction methods (32). This method is expected to provide an even more exhaustive list of metabolites. It is also expected that differences in lipid metabolite levels, including cholesterol, can be observed. Cholesterol modifies the hedgehog protein and is a precursor to certain hormones including ecdysone, a hormone related to polyphenism in Bicyclus and potentially involved in horn development (5). Another future method may be to utilize GC/MS on a global scale to identify cholesterol and other lipid metabolites.

In addition, our current untargeted results may be used to generate a hypothesis utilizing targeted LC/MS. This way, future researchers could focus on specific types of metabolites and pathways that may contribute to horn development. Metabolites may also be mapped onto biochemical and developmental pathways. 


\section{Chapter Two: Exploring the link between PI4P and horn development}

\section{Background}

In conjunction with the curation of an extensive list of metabolites present at a given developmental stage, a specific metabolite involved in the Hedgehog pathway was also studied. As mentioned, the Hedgehog pathway is a highly conserved developmental pathway that may have been co-opted into suppressing horn development in 0 . taurus exposed to low nutrient conditions. The metabolite in question, phosphatidylinositol-4-phosphate (PI4P), belongs to a group of signaling molecules known as phosphatidylinositol lipids (PIs). Pls are involved in many aspects of cellular regulation, with several playing critical roles in cell migration, actin formation, and lipid homeostasis (19).

Within the PI pathway, PI4P plays a role as a precursor to other phosphatidylinositol phosphates (PIPs) such as PI 3,4,5-trisphosphate (PIP3) which is involved in AKT signaling. AKT is a signaling cascade that stimulates glycogen synthesis when activated. In addition, it plays several roles in insulin signaling including phosphorylation and inhibition of Foxo, which is involved in the mediation of horn development (33).

In addition to this precursor role, PI4P also functions as a signaling molecule in the Hedgehog signaling pathway (34). When PI4P acts as a signaling molecule, it modulates Hh signaling by playing a critical role between receptor for the Hh ligand, Patched (Ptc) and the Gcoupled protein receptor, Smoothened (Smo), both of which are part of Hh signaling $(19,34)$. It is well known that these proteins aid in either regulating or transducing the signal from the $\mathrm{Hh}$ protein. In the presence of the Hh protein, Ptc releases its inhibition on Smo, leading to the activation of Hedgehog target genes (34). 
Researchers speculate that it is unlikely that Ptc interacts with Smo directly and instead, PI4P activates Smo via phosphorylation (36) (fig. 9). It has also been uncovered that the deactivation of Ptc leads to accumulated levels of PI4P, which further indicates that PI4P and Smo are positioned downstream of Ptc (19). In addition, research has found that the activation of Smo signaling activity is dependent on heightened levels of PI4P (35). Taken together, this establishes that the presence of PI4P leads to Smo activation. It has been found that while increased levels of PI4P promote Hh signaling, decreased levels inhibit it (36).

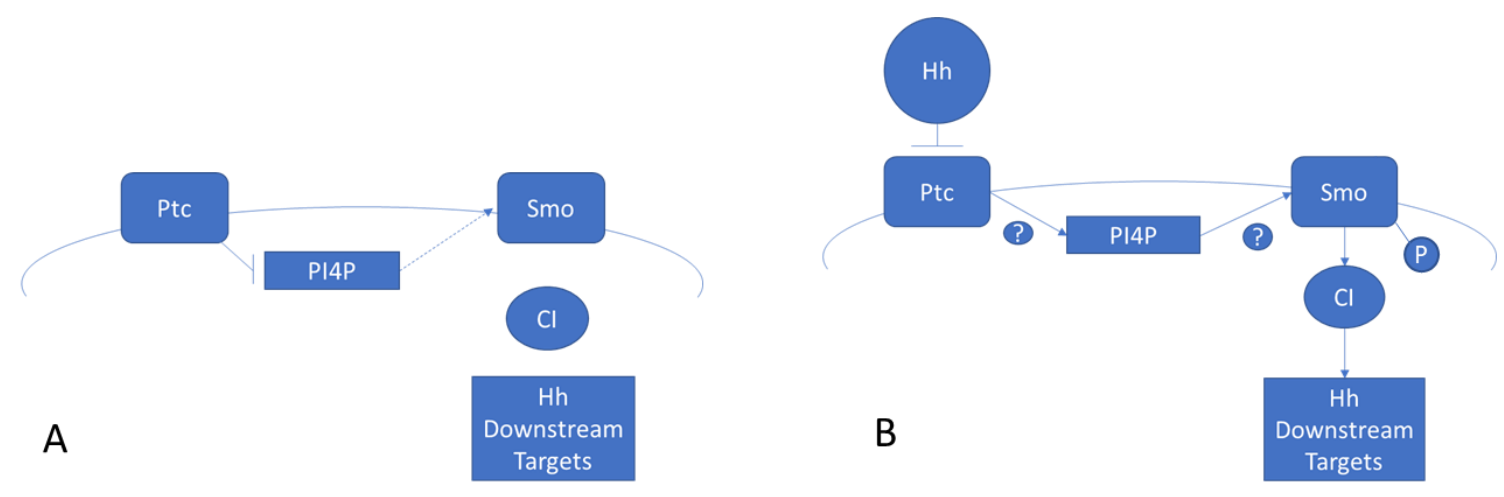

Figure 9: The effect of the Hh protein on PI4P and the activation of the Hedgehog pathway. A) In the absence of the Hh ligand, Ptc inhibits Smo via PI4P inhibition. Hh target genes not transcribed. Cubitus interruptus $(\mathrm{Cl})$ acts as a transcription factor

\section{Objectives}

Within the PI pathway, phosphatidylinositol can undergo a number of phosphorylations and dephosphorylations via different phosphatases and kinases to generate various phosphatidylinositol phosphates (PIPs). One crucial step of the PI pathway is the generation of PI4P via the phosphorylation of PI at the D-4 position of the inositol ring by PI4KIII $\alpha$ (fig. 10 (Image adopted from Tan 2014)). 


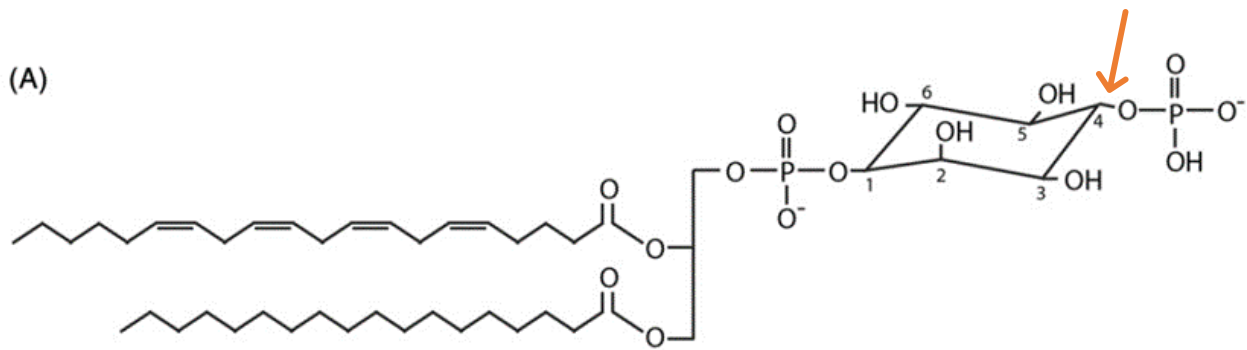

Figure 10. PI4P is a phospholipid that is metabolized from PI through the phosphorylation of the fourth position on the inositol ring. (adapted from Tan 2014)

High levels of PI4P can be detected near the plasma membrane as well as near all intercellular membranes to some degree. Different PI4Ks generate different pools of PI4P that are then utilized for different functions (19).

This study focuses on the significance of PI4P to the Hedgehog pathway by targeting genes involved in PI4P's phosphorylation (formation) and dephosphorylation (degeneration) through RNAi. In order to target the metabolism of this lipid metabolite, a gene knockdown has been conducted on phosphatidylinositol 4-kinase III alpha (PI4KIII $\alpha$ ), the kinase that produces pools of PI4P for use in Hedgehog signaling (Fig. 11).
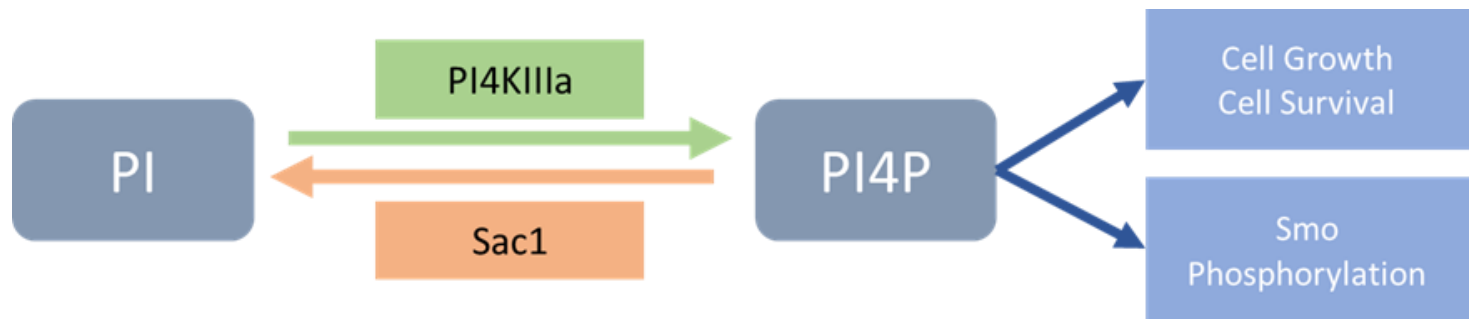

Figure 11: PI is phosphorylated into PI4P by PI4KIIl $\alpha$, which can then be dephosphorylated by Sac1 back into PI. $\mathrm{PI} 4 \mathrm{P}$ is utilized as a precursor for lipids involved in cell growth and survival or used for Smo phosphorylation in Hh signaling. 
Through studying the gene coding for this kinase, insight may be gained regarding how PI4P has been co-opted with the Hedgehog signaling pathways to play a role in the polyphenic development of horns.

\section{Hypothesis}

The knockdown of PI4KIII $\alpha$ is hypothesized to affect horn size. Our results are expected to phenocopy the result of a previous Hh knockdown, in which Hh and smo RNAi resulted in large horns developing in small males under low nutrient conditions (8).

As mentioned in the introduction, beetles in natural populations exhibit normal distribution in thorax width and a bimodal distribution in horn size. An increase in horn size was expected to occur in PI4KIII $\alpha$ RNAi beetles, while thorax width was expected to remain consistent with control animals.

\section{Materials and methods}

\section{Genes}

The PI4KIIl $\alpha$ gene in $O$. taurus is $6001 \mathrm{bp}$. The gene was obtained through the 0 . taurus Genome Project (part of the I5K Project) (37) and compared against PI4KIIl $\alpha$ in other organisms using Blast, CLUSTAL Omega (1.2.4) for sequence alignments, and Clustal 2.1 for Percent Identity Matrices. This was conducted in order to determine the selected gene's identity as the PI4KIIl $\alpha$. First, two superfamilies within our selected gene were compared against the same superfamilly in five different insects. These include Tribolium castaneum, a red flour beetle (beetle model organism), Oryctes borbonicus, another dung beetle, Drosophila melanogaster, a fruit fly (insect model organism), and Bicyclus anynana, a butterfly mentioned above that falls between beetles and D. melanogaster phylogenetically. The first domain, the PI3K $\alpha$ 
superfamily, was shown to have high similarity when compared between the above organisms and 0 . taurus (fig. 12a). The second compared domain, the PI4Kc III alpha superfamily, displayed high similarity as well (fig. 12b).

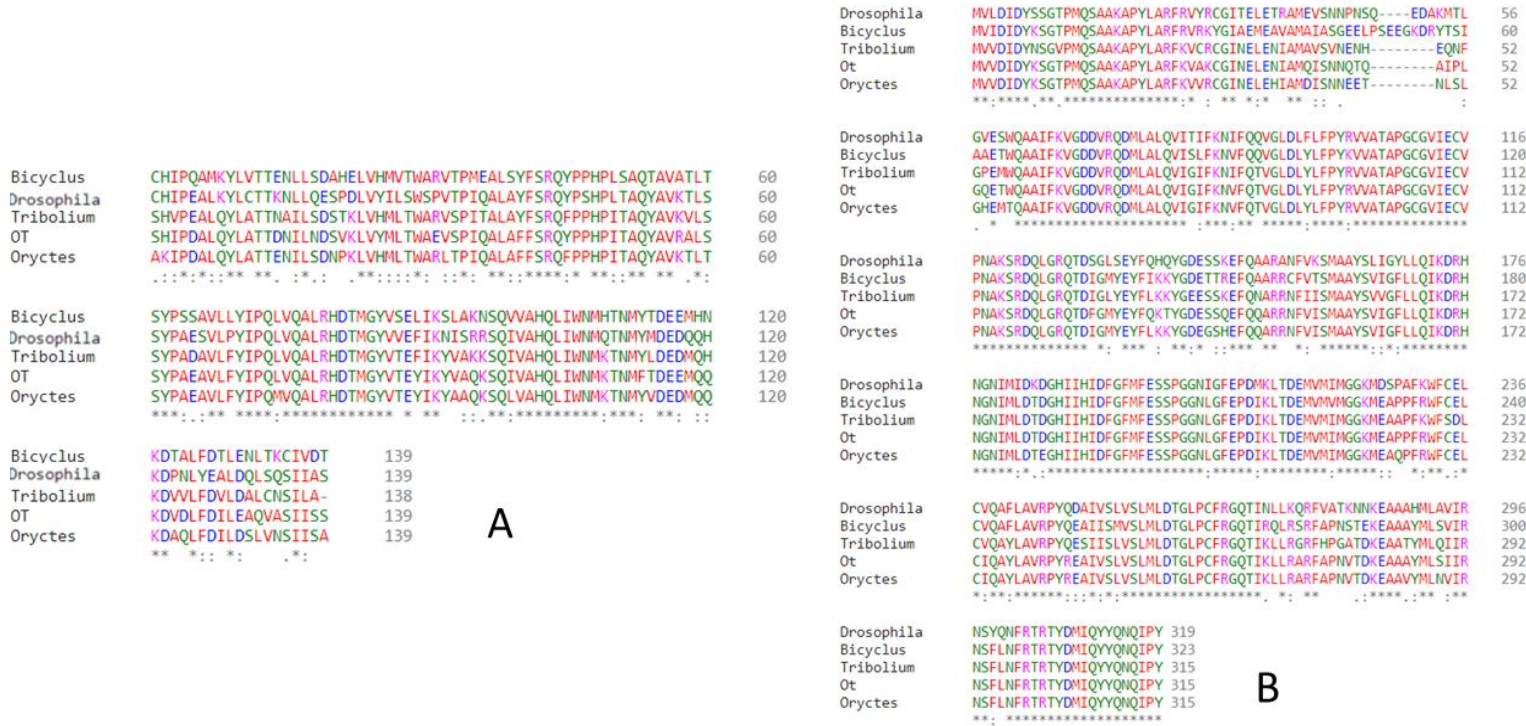

Figure 12. Sequence data from two superfamilies within the PI4KIIl $\alpha$ gene comparing five organisms. A) PI3Ka domain. B) PI4Kc III alpha. Results were used to confirm our gene of interest.

The entire sequence for PI4KIII $\alpha$ was then compared between $O$. taurus and $D$. melanogaster using BlastX and found to have an overall sequence identity of $55.11 \%$. These results suggest that the gene in question likely to be the PI4KIII $\alpha$ ortholog in 0 . taurus.

Gene fragments for PI4KIII $\alpha$ and were amplified and cloned into a vector using StrataClone PCR Cloning Kit (Agilent Technologies Cedar Creek, TX). The GeneJET Plasmid MiniPrep kit (Thermo Fisher Scientific Pittsburgh, PA) was used for plasmid preparation prior to sequencing. Two-thirds of the PI4KIII $\alpha$ gene was able to be confirmed by Sanger sequencing, 
and a gene specific fragment was located (fig. 13). Due to a computer malfunction, the resulting sequences were unable to be located.

The gene-specific fragment for PI4KIIl $\alpha$ was amplified and cloned into a vector using the StrataClone PCR Cloning Kit and the plasmid was prepared using the GeneJET Plasmid MiniPrep Kit. T3 and T7 RNA polymerases were utilized in the in vitro transcription to generate doublestranded RNA (MegaScript RNAi Kit, Thermo Fisher Scientific). Specifically, single stranded RNA

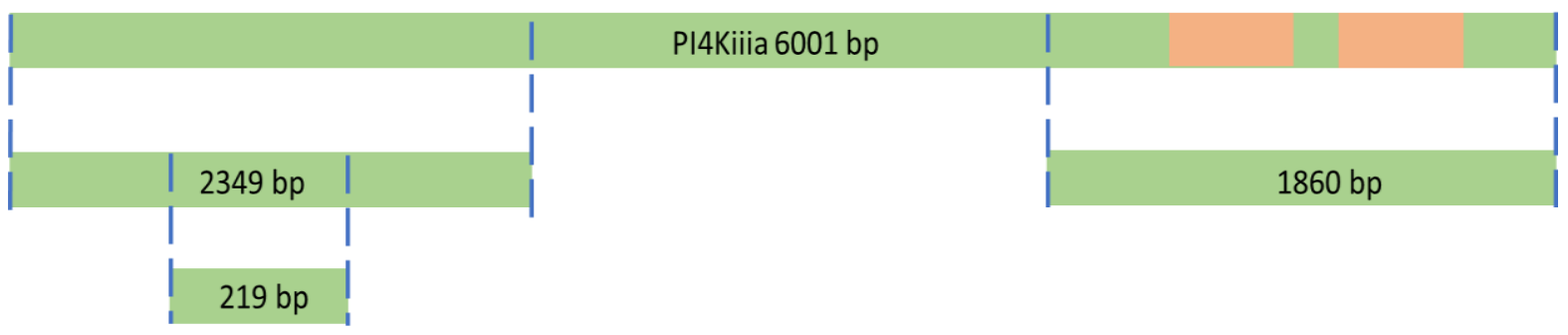

Figure 13: Visualization of the gene PI4KIII $\alpha$. Two segments of the gene were able to be isolated in 0 . taurus. The gene specific region used for dsRNA synthesis was around 219 base pairs long. Orange bars represent conserved domains PI3K $\alpha$ and PI4Kc III alpha, respectively.

was generated and mixed by matched amounts to generate double-stranded RNA (dsRNA). It was then heated and the gradually cooled down. dsRNA was stored in $-20^{\circ} \mathrm{C}$.

Beetle larvae were injected with $1 \mu \mathrm{g} / 3 \mu \mathrm{l}$ of dsRNA. Larvae were then observed, and their mass was measured at pre-pupa two stage. Beetles were then collected upon death or maturity and preserved in $70 \% \mathrm{EtOH}$.

Animals images were obtained using a Leica microscope with a Scion camera. Measurements were obtained using imageJ software (38). Horns were measured from the corner of the eye to the tip of the horn and means were calculated. The thorax was measured 
across the widest part (fig. 14). These measurements were compared between PI4KIII $\alpha$ animals and control animals.
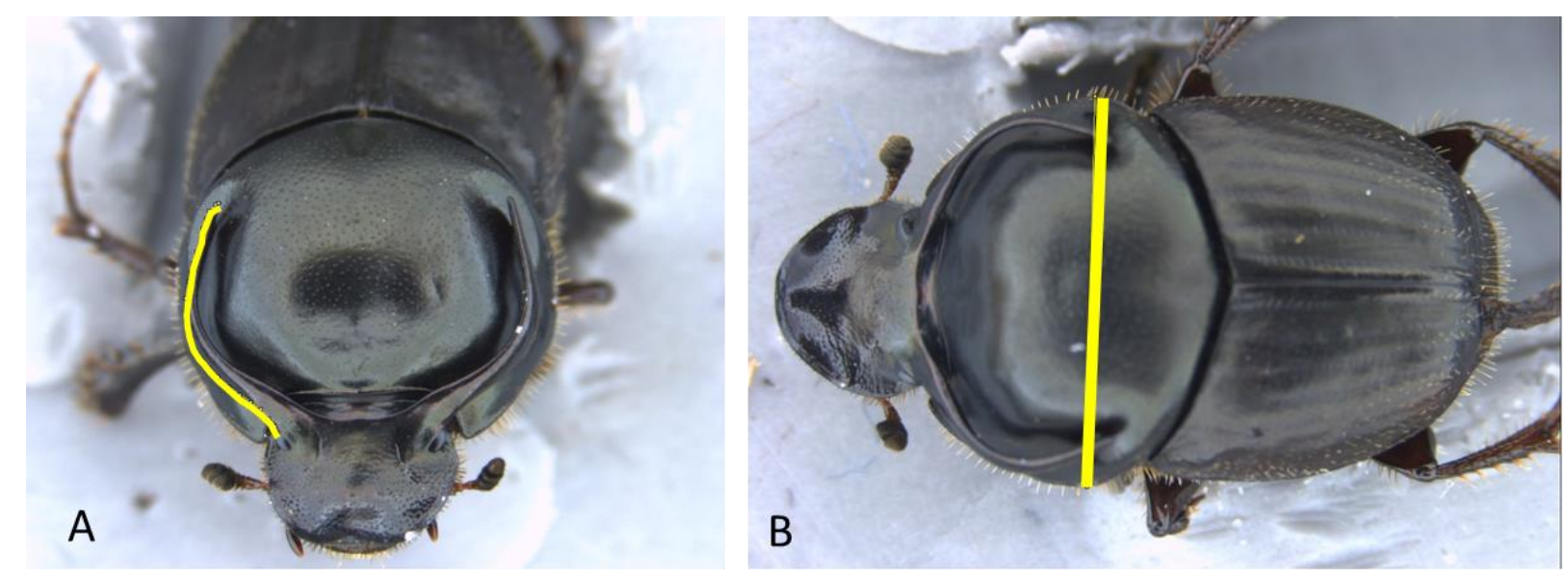

Figure 14: Horn and thorax measurements. A) Horns are measured one at a time and averaged to produce horn size. B) Thorax images are taken from the top down and measured at the widest part of the thorax; this measurement is used as the thorax width.

\section{Statistics}

We compared horn sizes and thorax widths, quantified horn shapes, and took a qualitative approach to analyzing phenotypes between the control $(n=37)$ and PI4KIII $\alpha$ knockdown ( $n=25)$ groups. JMP was utilized to determine the difference in mean horn size, fit a curve to determine different groups, and compare horn shape by analyzing horn ratio (40).

The Kuiper test is one-dimensional and took into account horn size independent of thorax width. This test differs from similar tests (such as the Kolmogorov-Smirnov test) as it detects differences in the tails of the distribution instead of just the center and, thus, is more sensitive The Kuiper test showed that there was significance in distribution with a p-value of 0.015 . 
Next, we fit a curve to horn size vs thorax width distribution for both groups.

Interestingly, different models fit better depending on the dataset (fig. 15). While control animals fit 3 Parameters Logistic (Logistic 3P), PI4KIIl $\alpha$ fit a linear model.
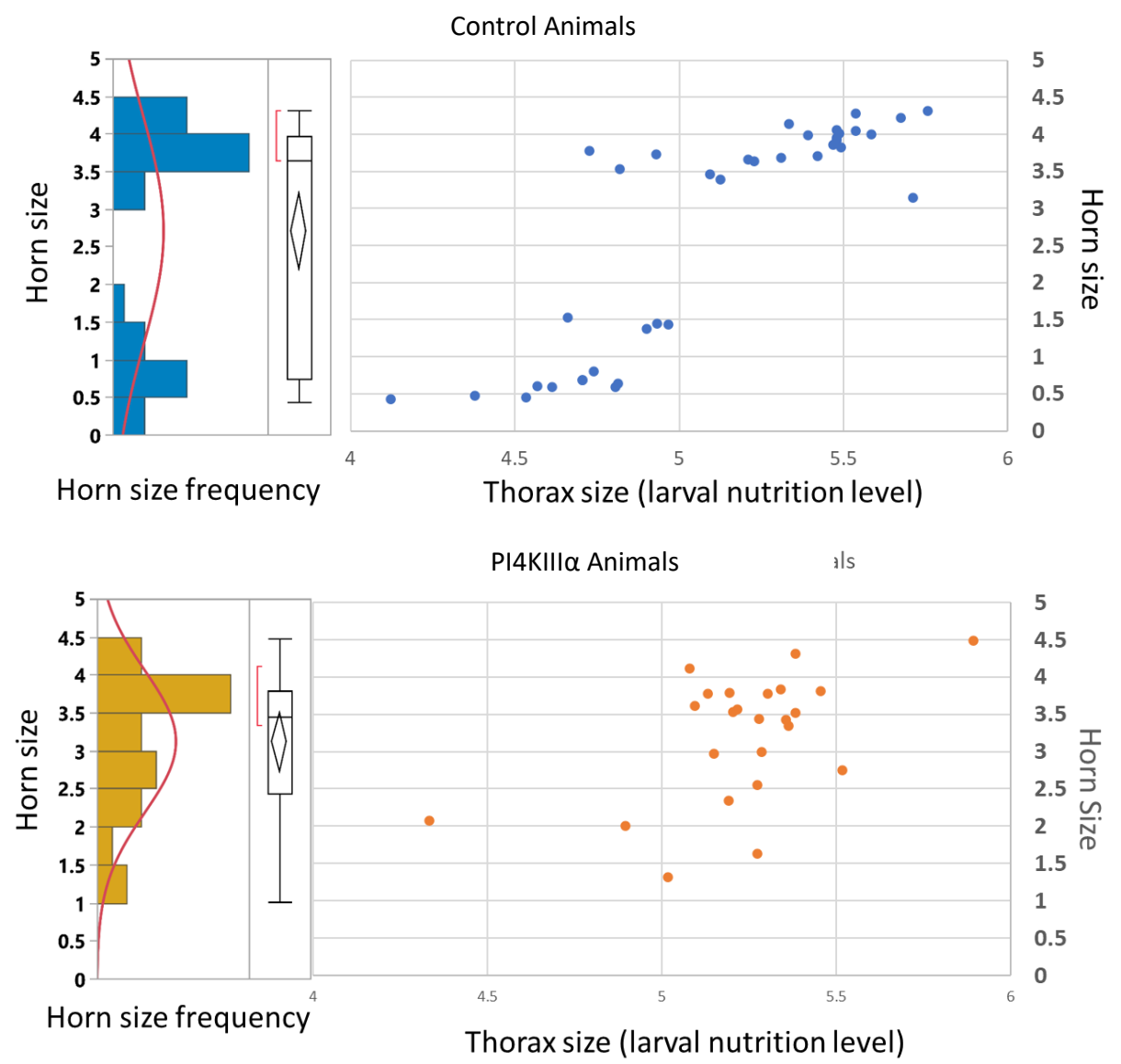

Figure 15: Distribution of control and PI4KIIl $\alpha$ animals. Control animals fit a logistic 3P curve and have a bimodal distribution while PI4KIII $\alpha$ fit a linear curve and have a unimodal distribution

Utilizing AICc notations, we were able to determine which curve best fit each group (fig. 16). These fits were further supported by statiscially significant perameters for each fit. The distribution of control was significant for all Logistic 3P perameters including growth rate $(p=$ 0.0006), inflection point $(p<0.0001)$, and asmyptote $(p<0.0001)$. PI4KIIl $\alpha$ showed significance 
in the parameter for slope $(p=0.0171)$ signifying a linear distribution. There is no overall $p$ value that correlates testing best fit.

\begin{tabular}{|l|l|l|}
\hline & Control AICc & Pi4kiiia AICc \\
\hline Linear & 94.303064 & 57.194438 \\
\hline 3P & 89.813808 & 60.009038 \\
\hline 4P & 92.124765 & 63.070695 \\
\hline
\end{tabular}

Figure 16: AICc values for control and PI4KIII $\alpha$ distributions. Lower numbers equate to better fits. While control best fits logistic 3P PI4KIIl $\alpha$ best fits a linear model.

While horn size was significantly different between the control and PI4KIIla groups, thorax width was not. We applied the Wilcoxon test due to one of the groups lacking normality and determined that there was no significant difference between the two groups $(p=0.6564)$.

In addition to studying horn size and its relation to thorax width, quantitative horn shapes were also analyzed. For this project, a measurement system was developed that takes the width between the tips of the two horns and divides them by the widest part at the center of the horns (fig. 17). We hypothesized that horns in PI4KIIl $\alpha$ beetles would have an overall greater ratio. 


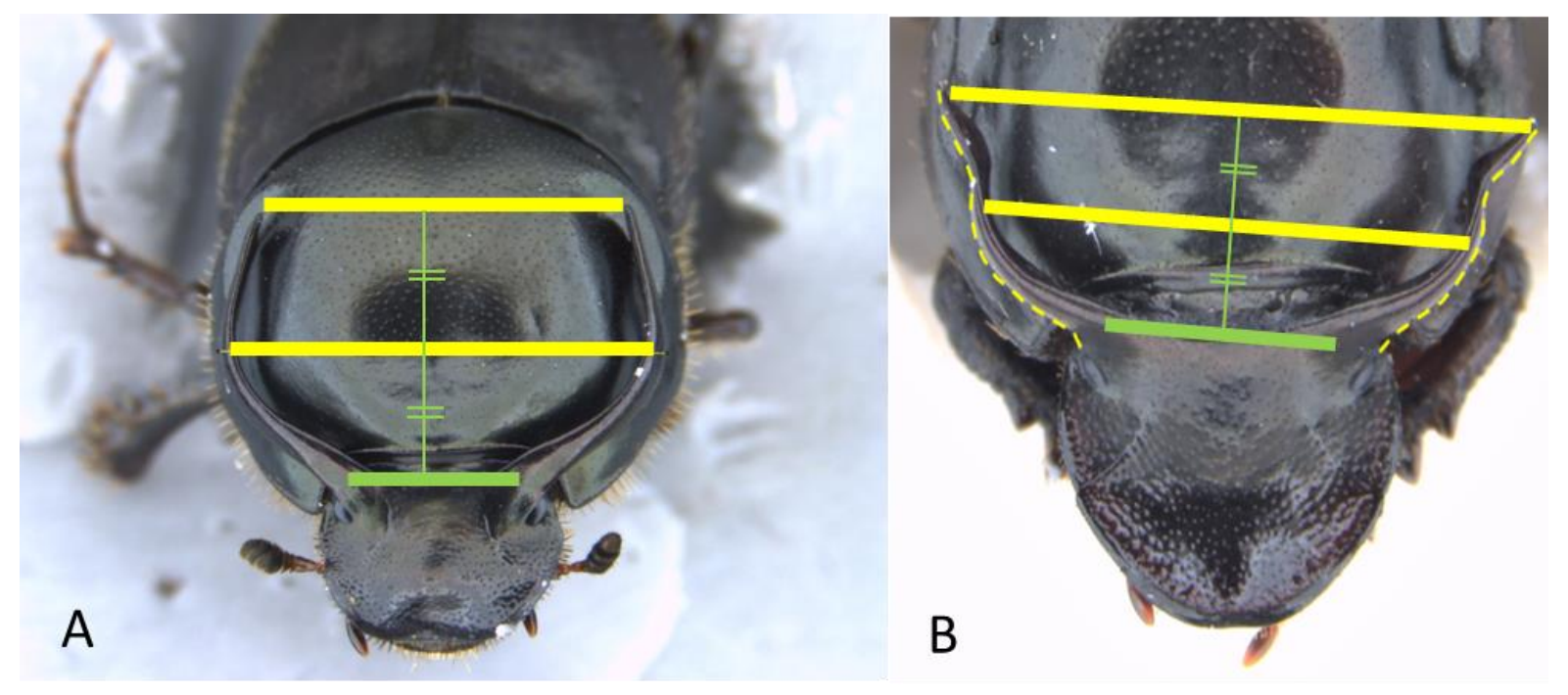

Figure 17: beetle horns were measured from tip to tip and center to center on A) control animals and B) PI4KIIl $\alpha$ animals

Statistical testing was also conducted on horn ratios to determine differences between control and treatment (fig. 18). Standard deviation for control was 0.070747 and it increased to 0.154689 in the PI4KIII $\alpha$ treatment group.

Horn Ratio Counts for Control and PI4KIII $\alpha$
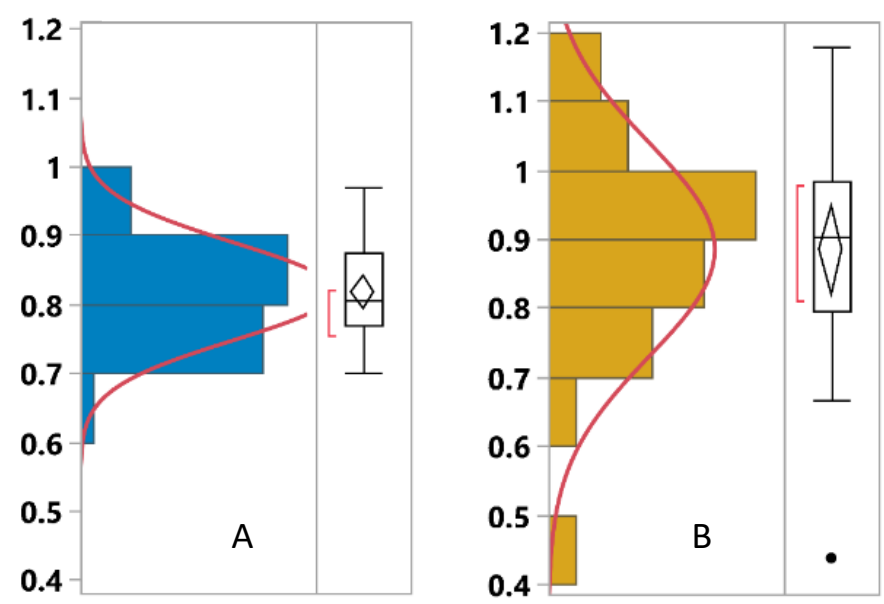

Figure 18: Horn ratio measurements for (a) control and (b) PI4KIII $\alpha$ knockdown animals. Data for PI4KIII $\alpha$ animals have greater variability than did data for control animals. 
We were able to determine that there was a significant difference in the variance between the two groups by applying a Levene test $(p$-value $=0.0011)$. Due to this unequal variance and normal distribution, we utilized Welch Anova testing and determined that the probability of the differences in means being due to chance ( $p$-value) was 0.0540.

We then aimed to compare our phenotype data with that of a Smoothened (Smo) knockdown. We utilized a Cochran-Mantel-Haenszel $(\mathrm{CMH})$ test to determine if the percent of beetles in each group groups (control, PI4KIIl $\alpha$, and Smo) shifted when compared to phenotype severity (none, moderate, severe) (41). Beetles are considered to have a moderate horn phenotype if one horn has a different than expected shape (kinked, curled under (fig. 20e)), both horns have an intermediate size (between $2 \mathrm{~mm}$ and $3 \mathrm{~mm}$ ), or both horns have a tip to center ratio of approximately 1. If horns display two or more of the above phenotypes (such as both horns being curled, or one horn turning out and both horns having an intermediate size) then they are considered severe

The $\mathrm{CMH}$ test displays the percent of beetles from each treatment group divided into none, moderate, and severe horn phenotype groups (table 2, fig. 19). The CMH test also generates two statistics that contribute to the significance of differences. The first is non-zero correlation, which ranks beetle groups in terms of severity and was shown to be significant $(p=$ 0.0481). The second is Row Mean Scores Difference, which demonstrates the shift in mean among the different severity categories and was also significant $(p<0.0001)$. 
Table 2: Horn phenotype data for Control, PI4KIII $\alpha$, and Smoothend animals in percentages. Beetles are considered to have a moderate horn phenotype if one horn has a different than expected shape (kinked, curled under), horns have an intermediate size, or horns have a tip to center ratio 1 . If horns display two or more of the above phenotypes (such as both horns being curled, one horn turning out and both horns having an intermediate size) then they are considered severe. develop. Statistics show that type of beetle is significantly correlated with phenotype counts.

\begin{tabular}{|l|l|l|l|}
\hline & None & Moderate & Severe \\
\hline Control & 94.59 & 5.41 & 0.00 \\
\hline PI4KIII & 44.44 & 33.33 & 22.22 \\
\hline Smo & 72.73 & 21.21 & 6.06 \\
\hline
\end{tabular}

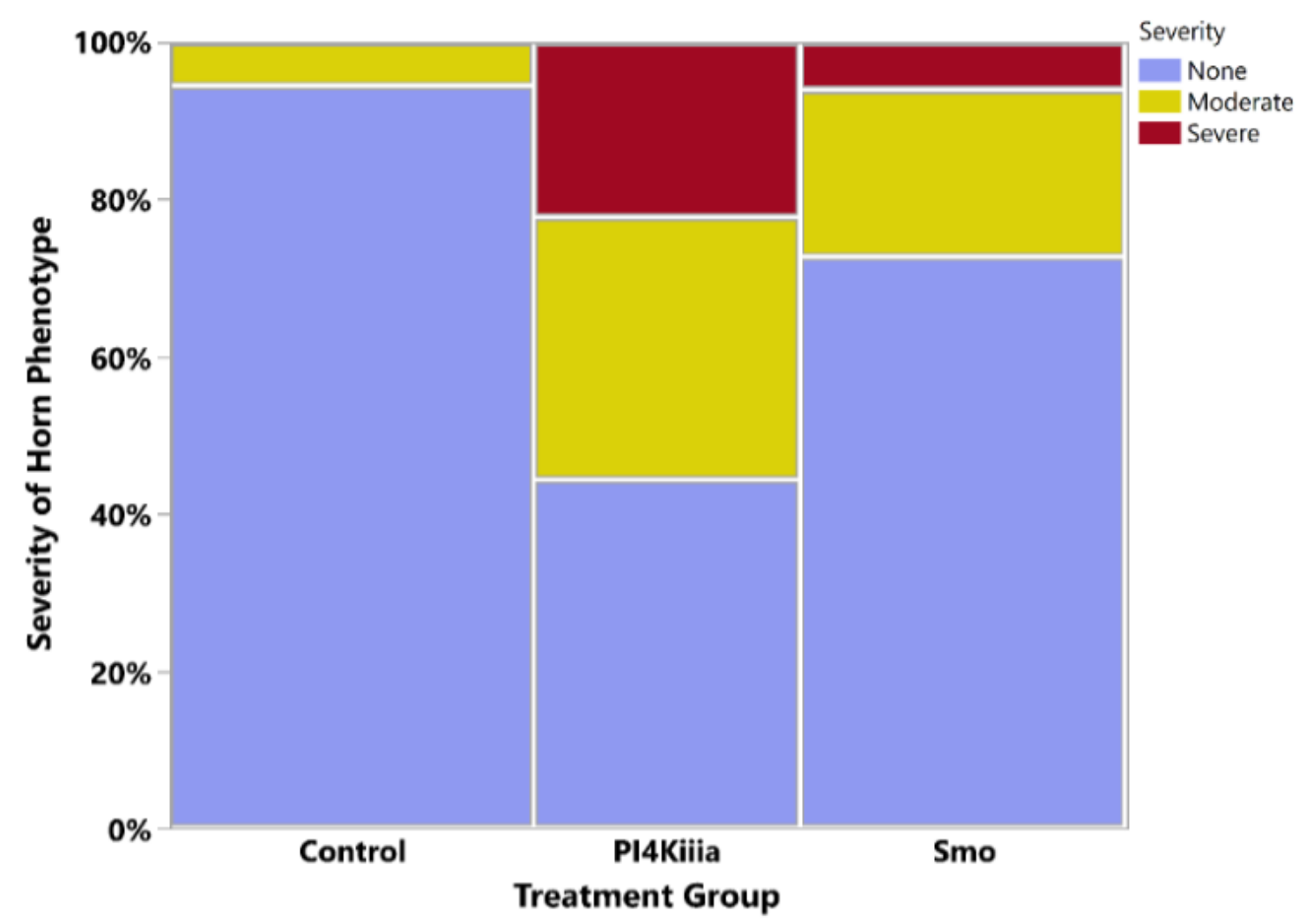

Figure 19: Mosaic plot displaying the percent of none, moderate and severe horn phenotypes seen in each group. PI4KIII $\alpha$ has the highest percentage of moderate and severe phenotypes. 
In addition to horn phenotype similarities between PI4KIII $\alpha$ and Smo beetles, a number of beetles also developed similar elytra (forewing) malformation. This may be due to Smo's role in appendage formation during pupal development (8). Elytra phenotype data counts comparing control, PI4KIII $\alpha$, and Smoothened animals. In order to better compare these phenotypes, we placed beetles into one of the three categories: none, moderate, and severe. Beetle elytra were considered moderate when they show less than $50 \%$ malformation. Severe elytra phenotypes include animals with greater than $50 \%$ malformation or elytra are curled under or failed to develop.

The $\mathrm{CMH}$ test was also conducted on the elytra phenotype data and a mosaic plot was generated (table 3, fig. 20). Again, this test was utilized to determine if there is a significant shift the percent of beetles in each category. As with horn data, the non-zero correlation was significant $(p<0.0001)$, along with the row means score difference $(p<0.0001)$.

Table 3: Elytra phenotype data presented in percentages comparing Control, PI4KIIl $\alpha$, and Smoothened animals. Elytra phenotypes are considered none with no malformation and moderate when horns show less than $<50 \%$ damage. Severe elytra phenotypes include animals with $>50 \%$ damage or elytra that are curled under or failed to develop. Statistics show that type of beetle is significantly correlated with phenotype counts.

\begin{tabular}{|l|l|l|l|}
\hline & None & Moderate & Severe \\
\hline Control & 83.78 & 10.81 & 5.41 \\
\hline PI4KIII & 52.00 & 20.00 & 28.00 \\
\hline Smo & 6.90 & 3.45 & 89.66 \\
\hline
\end{tabular}




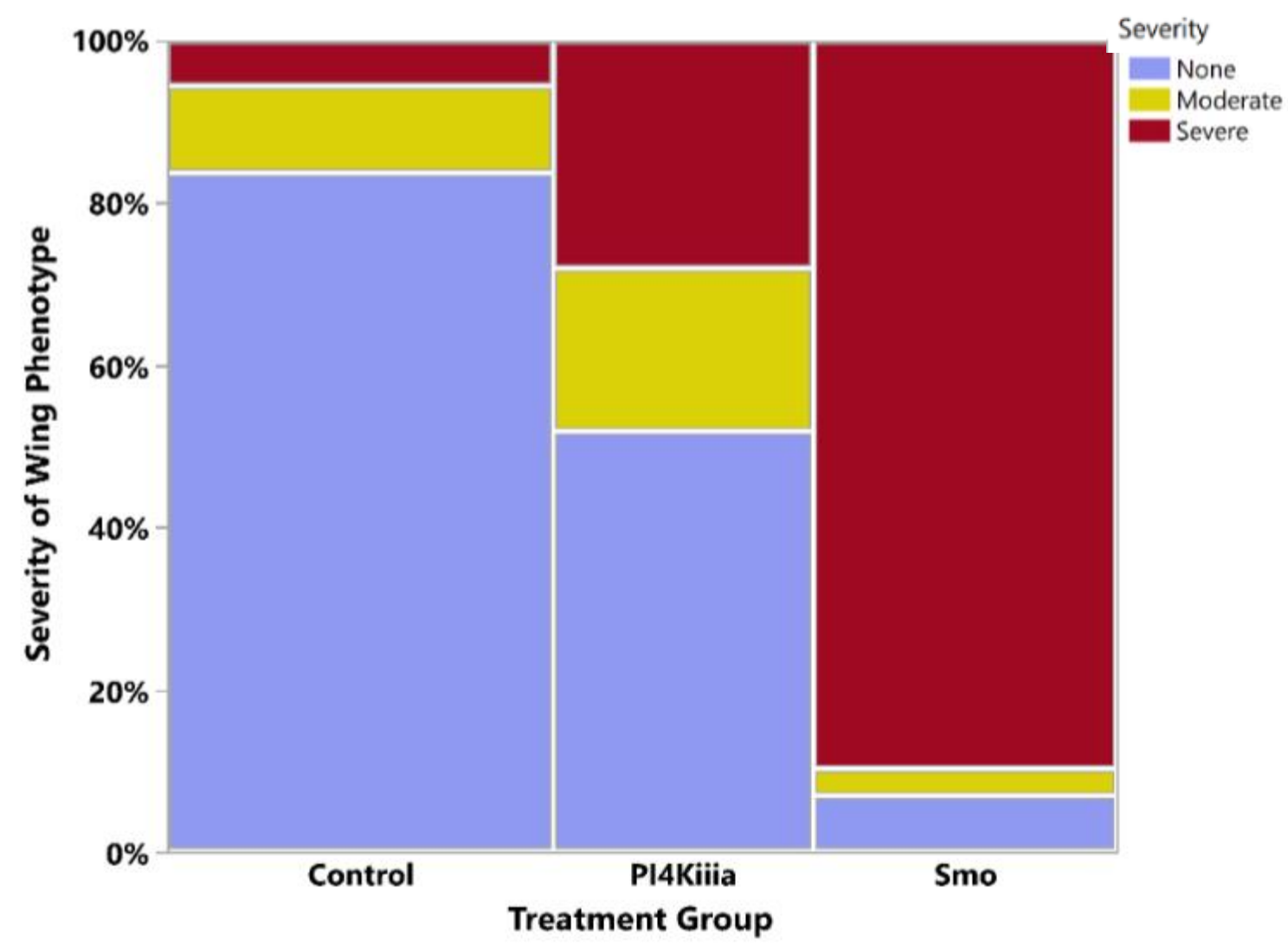

Figure 20: Mosaic plot displaying the percent of none, moderate and severe elytra phenotypes seen in each group. $89.66 \%$ of Smo animals show severe elytra malformation.

Results

Through the Kuiper test, we were able to determine that the mean horn size of PI4KIII $\alpha$ animals is significantly greater than the horn size for control individuals. For control individuals, the curve that fit best was 3 Parameters Logistic (Logistic 3P), while the best fit for PI4KIII $\alpha$ was a linear model (fig. 14). The information that different fits are better for different sets of data show that data distribution is dramatically different between the control and PI4KIII $\alpha$ RNAi treatments. 


\section{Horn Width Ratio}

By comparing horn width ratio, we were able to quantify changes in horn shape due to the PI4KIII $\alpha$ knockdown. We determined that PI4KIII $\alpha$ horn ratios had a higher variance than control animals. In addition, the probability of the differences in horn ratio means not being due to chance was $94.6 \%$.

\section{Comparison to smoothened}

In a study by Kijimoto et al., RNAi was conducted on multiple components of the Hedgehog signaling pathway to determine the role they play in the development of horns in $O$. taurus (8). Our PI4KIII $\alpha$ knockdown beetles appeared to show phenotypic similarities to knockdowns of Smoothened (Smo) previously described by Kijimoto et al. (8). As with PI4KIII $\alpha$,

Smo knockdown individuals show increased horn size regardless of thorax width (fig. 21)

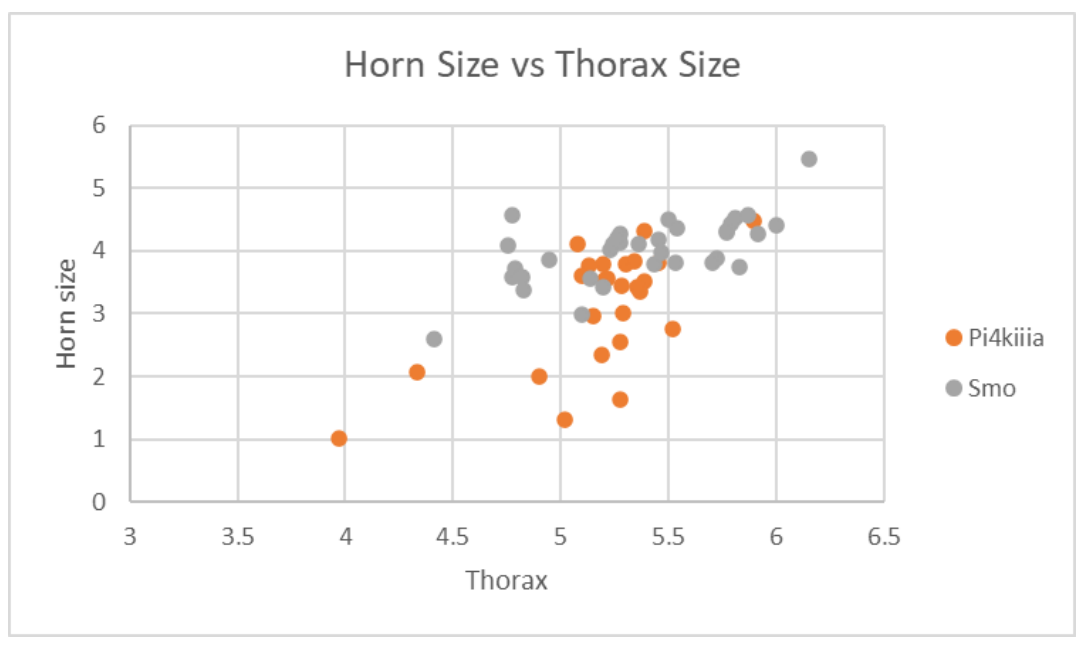

Figure 21. PI4KIII $\alpha$ animal measurements compared to Smo measurements. Both show increased horn size in small animals while thorax width is maintained 


\section{Phenotype Categorical Data}

In order to compare Smo, PI4KIII $\alpha$, and control beetles, a quantitative phenotypic approach was taken. We conducted a $\mathrm{CMH}$ test in order to compare these variables and determine if treatment group was correlated with severity of phenotype. Results show that the type of beetle knockdown is a significant predictor of both horn and elytra malformation severity. In addition, we were also able to determine that beetle groups could be ranked in terms of severity of phenotype. While horn data appeared to ranked control as being the least severe and PI4KIIl $\alpha$ being the most severe, elytra data seemed to rank control being the least severe and Smo being the most severe.

\section{Other Notable phenotypes}

PI4KIII $\alpha$ beetles exhibit a number of different phenotypes that are not seen in control individuals. Horns are outlined in yellow to display the diversity of phenotypes (fig. 22). Beetles with smaller bodies tended to show a wider range of horn phenotypes than large beetles. 

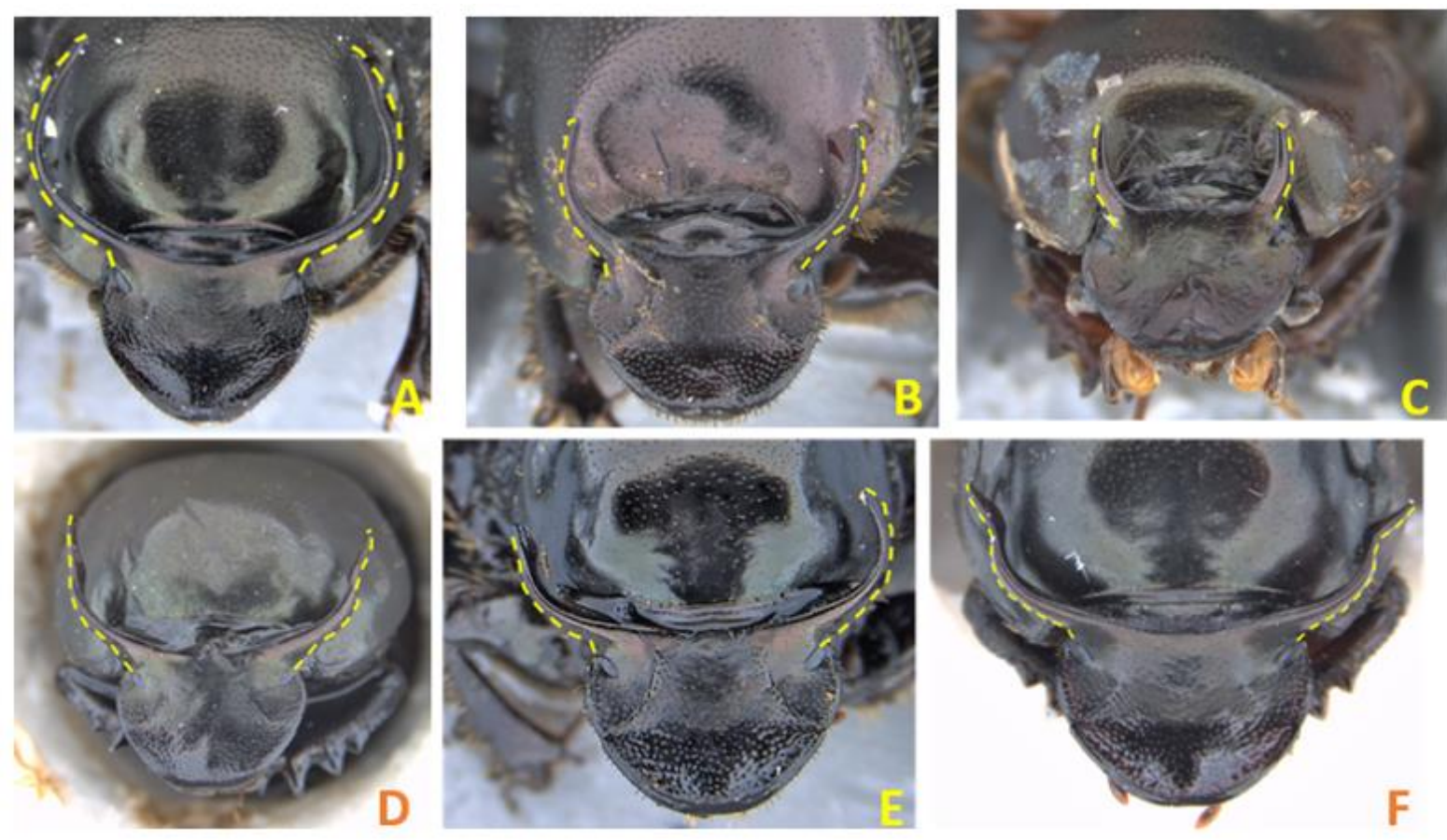

Image 22: Comparison of PI4Kiiia knockdown beetle horn phenotypes. A) A control beetle with a normal horn phenotype. B, C) Beetles with intermediate-sized horns and a moderate phenotype. D) A beetle with severe horn phenotype where horns kink at the tips and point upward E) A beetle with a severe horn phenotype where horns are an intermediate size and one horn turns outward. F) A beetle with horns that kink at the tips and point outward.

\section{Discussion}

By conducting a PI4KIII $\alpha$ knockdown, a better understanding of how this kinase affects the development of horns in $O$. taurus has been gained. Data shows that while thorax width remains constant, horn size is increased in small animals. This may signify that while PI4P plays a role in determining horn size (potentially through the Hedgehog signaling pathway), it does not impact thorax width.

Surprisingly, this knockdown also dramatically changed the distribution from sigmoidal in control animals to linear in knockdown animals. This also supports the hypothesis that PI4KIII $\alpha$ RNAi alters horn size in beetles. This change from curve to line is reminiscent of the 
distribution of Smo knockdown beetles, supporting the hypothesis that PI4P and Smo may interact in the hedgehog pathway to suppress horn development in beetles under low nutrient conditions.

We compared phenotype severity among the PI4KIII $\alpha$, Smo, and control animals through the $\mathrm{CMH}$ test. This test provided evidence that a shift in both horn and wing severity occurred between the different treatment groups. If also provided evidence that groups could be ranked in terms of severity. For horn phenotypes, the control group appears to be the least severe followed by Smo and PI4KIII $\alpha$. This provides evidence that PI4KIIl $\alpha$ has a greater influence over the development of horn shapes than Smo. However, when elytra phenotypes are categorizes, control appeared to be the lest severe followed by PI4KIIl $\alpha$ and then Smo. This indicates that Smo plays a bigger role in the development of elytra than PI4KIII $\alpha$ does.

In addition, we developed a method for quantifying horn shape by dividing the space between horn tips by the space between the center of the horns. Results indicated a low probability that changes in shape were due to chance $(p=0.054)$; a greater sample size may lower that $p$ value.

Animals also developed a number of phenotypes not seen in control animals. These include intermediate sized horns, horns where tips kink or turn out, and horns that are nearly parallel with each other. This indicates that PI4KIII $\alpha$ is not only related to horn size but may be related horn shape. This may also imply that the Hedgehog pathway not only works to suppress horn size, but also determine horn shape in small individuals. This could potententailly be 
caused by an increse in the Headgehog signaling pathway in small beetles contributing to horn shape as well through axis parerning.

\section{Future Plans}

In order to better understand how a knockdown of PI4KIII $\alpha$ affects PI4P and other PIs, a targeted LC/MS approach can be taken. Detecting Cubitus interruptus (which is the most downstream component of the Hh signaling pathway (refer to fig. 8)) after a PI4KIII $\alpha$ knockdown through immunostaining is another method that can be taken (39). Staining for the active form of this protein will test whether Hh signaling is also activated. qPCR may also be utilized to evaluate RNAi and see if the expression level of Hh signaling genes is altered by knocking down PI4KIII $\alpha$. Along with PI4KIIl $\alpha$, the phosphatase Sac1, which dephosphorylates PI4P into PI, could be targeted to determine how the repression of degradation of PI4P influences horn development. 


\section{Bibliography}

1) Fusco, G., and Alessandro M. 2010. Phenotypic Plasticity in Development and Evolution: Facts and Concepts. Philosophical Transactions of the Royal Society B: Biological Sciences. vol. 365, no. 1540, pp. 547-556., doi:10.1098/rstb.2009.0267.

2) Pigliucci, M. 2006. Phenotypic Plasticity and Evolution by Genetic Assimilation. Journal of Experimental Biology, vol. 209, no. 12, pp. 2362-2367., doi:10.1242/jeb.02070.

3) Lea, A., Tung, J., Archie, E., Alberts, S., 2017. Developmental plasticity: Bridging research in evolution and human health, Evolution, Medicine, and Public Health, Vol. 2017, no. 1, Pages 162-175

4) Hosseini, S., Brenig, B., Tetens, J., Sharifi, A., 2018 Phenotypic Plasticity Induced Using High Ambient Temperature during Embryogenesis in Domesticated Zebrafish, Danio Rerio. Reproduction in Domestic Animals, vol. 54, no. 3, pp. 435-444., doi:10.1111/rda.13382.

5) Bhardwaj S., Prudic K., Bear A., Dasgupta M., Wasik B., Tong X., Cheong W., Wenk MR., Monteiro A. 2017. Sex Differences in 20-Hydroxyecdysone Hormone Levels Control Sexual Dimorphism in Bicyclus Anynana Wing Patterns. Molecular Biology and Evolution, vol. 35, no. 2, pp. 465-472., doi:10.1093/molbev/msx301.

6) Miura, T. 2018 Juvenile Hormone as a Physiological Regulator Mediating Phenotypic Plasticity in Pancrustaceans. Development, Growth \& Differentiation, vol. 61, no. 1, pp. 85-96., doi:10.1111/dgd.12572.

7) Emlen D., Hunt J., Simmons L. 2005. Evolution of Sexual Dimorphism and Male Dimorphism in the Expression of Beetle Horns: Phylogenetic Evidence for Modularity, Evolutionary Lability, and Constraint. The American Naturalist, vol. 166, no. 4, doi: $10.2307 / 3473066$.

8) Kijimoto, T., and Moczek, A. 2016 Hedgehog Signaling Enables Nutrition-Responsive Inhibition of an Alternative Morph in a Polyphenic Beetle. Proceedings of the National Academy of Sciences, vol. 113, no. 21, pp. 5982-5987., doi:10.1073/pnas.1601505113

9) Moczek, A., 2005. The Evolution and Development of Novel Traits, or How Beetles Got Their Horns. BioScience, vol. 55, no. 11, p. 937., doi:10.1641/00063568(2005)055[0937:teadon]2.0.co;2.

10) True, R., and Carroll, S., 2002. Gene Co-Option in Physiological and Morphological Evolution. Annual Review of Cell and Developmental Biology, vol. 18, no. 1, pp. 53-80., doi:10.1146/annurev.cellbio.18.020402.140619. 
11) Ledón-Rettig C., Zattara E., Moczek A. 2017. Asymmetric Interactions between Doublesex and Tissue- and Sex-Specific Target Genes Mediate Sexual Dimorphism in Beetles. Nature Communications, vol. 8, no. 1, doi:10.1038/ncomms14593.

12) Price, D., Egizi, A., and Fonseca, D., 2015. The Ubiquity and Ancestry of Insect Doublesex. Scientific Reports, vol. 5, no. 1, doi:10.1038/srep13068.

13) Hedgehog Signaling Interactive Pathway. Cell Signaling Technology, www.cellsignal.com/contents/science-cst-pathways-stem-cell-markers/hedgehogsignaling-interactive-pathway/pathways-hedgehog.

14) Villarreal C., Darakananda K., Wang V., Jayaprakash P., Suzuki Y. 2015 Hedgehog Signaling Regulates Imaginal Cell Differentiation in a Basally Branching Holometabolous Insect. Developmental Biology, vol. 404, no. 2, pp. 125-135., doi:10.1016/j.ydbio.2015.05.020.

15) Grenier, J., Weatherbee S., Carroll S. 2010 From DNA to Diversity: Molecular Genetics and the Evolution of Animal Design. Blackwell

16) Varjosalo, M., and Taipale. J., 2008. Hedgehog: Functions and Mechanisms. Genes \& Development, vol. 22, no. 18, pp. 2454-2472., doi:10.1101/gad.1693608.

17) Casasa, S., Moczek, A ., 2018. Insulin Signalling's Role in Mediating Tissue-Specific Nutritional Plasticity and Robustness in the Horn-Polyphenic Beetle Onthophagus Taurus. Proceedings of the Royal Society B: Biological Sciences, vol. 285, no. 1893.

18) Fanos V., Antonucci R., Barberini L., Atzori L. 2012. Urinary Metabolomics in Newborns and Infants. Advances in Clinical Chemistry, vol. 58, pp. 193-223., doi:10.1016/b978-012-394383-5.00013-8.

19) Tan, J., and Brill. J., 2013 Cinderella Story: PI4P Goes from Precursor to Key Signaling Molecule. Critical Reviews in Biochemistry and Molecular Biology, vol. 49, no. 1, pp. 3358., doi:10.3109/10409238.2013.853024.

20) Schrimpe-Rutledge A., Codreanu S., Sherrod S., McLean J., 2016 Untargeted Metabolomics Strategies - Challenges and Emerging Directions. Journal of The American Society for Mass Spectrometry, vol. 27, no. 12, pp. 1897-1905., doi:10.1007/s13361-016-1469-y.

21) Gorrochategui E., Jaumot, J., Lacorte, S., Tauler, R., 2016. Data Analysis Strategies for Targeted and Untargeted LC-MS Metabolomic Studies: Overview and Workflow. TrAC Trends in Analytical Chemistry, vol. 82, pp. 425-442., doi:10.1016/j.trac.2016.07.004.

22) Thermo Compound Discoverer User Guide Software Version 3.1. 2019. Thermo Fisher Scientific Inc. XCALI-98120 Revision A. 
23) Kijimoto T., Snell-Rood E., 2014 Pespeni, M., Rocha, G., Kafadar, K., Moczek, A., The Nutritionally Responsive Transcriptome of the Polyphenic Beetle Onthophagus Taurus and the Importance of Sexual Dimorphism and Body Region. Proceedings of the Royal Society B: Biological Sciences, vol. 281, no. 1797.

24) Berg, J., Tymoczko J., Stryer L. 2002. The Glycolytic Pathway Is Tightly Controlled. Biochemistry. 5th edition. New York: W H Freeman; Section 16.2.

25) Krentz, A.J. 2000. Churchill's Pocketbook of Diabetes. Edinburgh: Churchill Livingstone.

26) Palou, A., and Bonet, M.1., 2007. Controlling Lipogenesis and Thermogenesis and the Use of Ergogenic Aids for Weight Control. Novel Food Ingredients for Weight Control, pp. 58103., doi:10.1533/9781845693114.1.58.

27) N-Acetyl-DL-glutamic acid, CID=185. National Center for Biotechnology Information. PubChem Database. https://pubchem.ncbi.nlm.nih.gov/compound/N-Acetyl-DL-glutamicacid

28) Metabocard for N-Acetyl-L-glutamic acid (HMDB0001138). Human Metabolome Database. The Metabolomics Innovation Centre. https://hmdb.ca/metabolites/HMDB0001138

29) Chou, C. 2013. Identification of the Pivotal Role of Glutamate in Enhancing Insect Cell Growth Using Factor Analysis. Cytotechnology, vol. 66, no. 5, pp. 853-860.

30) Villar, V.H., Merhi, F., Djavaheri-Mergny, M., Durán R.V. 2015. Glutaminolysis and autophagy in cancer. Autophagy, vol. 11, no. 8, pp. 1198-1208.

31) Metabocard for D-Xylonic acid (HMDB0059750). Human Metabolome Database. The Metabolomics Innovation Centre. https://hmdb.ca/metabolites/HMDB0059750 $\underline{\underline{30}}$

32) Bligh, E., and Dyer, W., 1959. A Rapid Method Of Total Lipid Extraction And Purification." Canadian Journal of Biochemistry and Physiology, vol. 37, no. 8, 1959, pp. 911-917., doi:10.1139/059-099.

33) Insulin Receptor Signaling. 2003. Cell Signaling Technology. Cell Signaling Technology, Inc. https://www.cellsignal.com/contents/science-cst-pathways-cellularmetabolism/insulin-receptor-signaling/pathways-irs

34) Arensdorf A., Marada S., Ogden S. 2015 Smoothened Regulation: A Tale of Two Signals. Trends in Pharmacological Sciences, vol. 37, no. 1, pp. 62-72., doi:10.1016/j.tips.2015.09.001.

35) Yavari A., Nagaraj R., Owusu-Ansah E., Folick A., Ngo K., Hillman T., Call G., Rohatgi R., Scott M., and Banerjee U. 2010 Role of Lipid Metabolism in Smoothened Derepression in Hedgehog Signaling. Developmental Cell, vol. 19, no. 1, pp. 54-65., doi:10.1016/j.devcel.2010.06.007. 
36) Jiang K., Liu Y., Fan J., Zhang, J., Li, X., Evers, M., Zhu, H., Jia, J., 2016. PI(4)P Promotes Phosphorylation and Conformational Change of Smoothened through Interaction with Its C-Terminal Tail. vol. 14, no. 2, doi:10.1371/journal.pbio.1002375.

37) The i5K Initiative: Advancing Arthropod Genomics for Knowledge, Human Health, Agriculture, and the Environment. Journal of Heredity, vol. 104, no. 5, 2013, pp. 595600., doi:10.1093/jhered/est050.

38) Schneider, C., Rasband, W., and Eliceiri, K., 2012. NIH Image to ImageJ: 25 years of image analysis. Nature methods, doi:10.3410/f.717951500.793456800

39) Méthot, N. and Basler, K., 2001 An absolute requirement for Cubitus interruptus in Hedgehog signaling. Development. vol. 128, pp. 733-742.

40) JMP®, Version Pro 14.0, SAS Institute Inc., Cary, NC, Copyright 2015; SAS®, Version 9.4, SAS Institute Inc., Cary, NC, Copyright 2002-2012.

41) Stokes, M. E., Davis, C.S., Koch, G. G., 2012. Categorical Data Analysis using SAS ®, Third Edition. Cary, NC: SAS Institute Inc. 